\title{
The Local Group on FIRE: dwarf galaxy populations across a suite of hydrodynamic simulations
}

\author{
Shea Garrison-Kimmel ${ }^{\odot},{ }^{1 \star} \dagger$ Philip F. Hopkins ${ }^{\oplus},{ }^{1}$ Andrew Wetzel ${ }^{\oplus},{ }^{1,2,3} \ddagger$ James \\ S. Bullock, ${ }^{4}$ Michael Boylan-Kolchin ${ }^{\oplus}{ }^{5}$ Dušan Kereš, ${ }^{6}$ \\ Claude-André Faucher-Giguère ${ }^{\oplus}, 7$ Kareem El-Badry ${ }^{\oplus}, 8$ Astrid Lamberts ${ }^{\oplus}, 1$ \\ Eliot Quataert ${ }^{8}$ and Robyn Sanderson ${ }^{\oplus 1}$ \\ ${ }^{1}$ TAPIR, Mailcode 350-17, California Institute of Technology, Pasadena, CA 91125, USA \\ ${ }^{2}$ The Observatories of the Carnegie Institution for Science, Pasadena, CA 91101, USA \\ ${ }^{3}$ Department of Physics, University of California, Davis, CA 95616, USA \\ ${ }^{4}$ Department of Physics and Astronomy, Center for Cosmology, University of California, Irvine, CA 92697, USA \\ ${ }^{5}$ Department of Astronomy, The University of Texas at Austin, 2515 Speedway, Stop C1400, Austin, TX 78712, USA \\ ${ }^{6}$ Department of Physics, Center for Astrophysics and Space Science, University of California at San Diego, 9500 Gilman Drive, La Jolla, CA 92093, USA \\ ${ }^{7}$ Department of Physics and Astronomy and CIERA, Northwestern University, 2145 Sheridan Road, Evanston, IL 60208, USA \\ ${ }^{8}$ Department of Astronomy and Theoretical Astrophysics Center, University of California Berkeley, Berkeley, CA 94720, USA
}

Accepted 2019 May 9. Received 2019 March 29; in original form 2018 June 8

\begin{abstract}
We present a new set of high-resolution hydrodynamic cosmological zoom-in simulations that apply the Feedback In Realistic Environments physics to both Local Group (LG)-like and isolated Milky Way (MW)-like volumes (10 host systems in total with a baryonic particle mass $\simeq 3500-7000 \mathrm{M}_{\odot}$ ). We study the stellar mass functions, circular velocity or mass profiles, and velocity dispersions of the dwarf galaxy populations. The simulations reproduce the stellar mass function and central densities of MW satellite dwarfs for $M_{*} \geq 10^{5.5} \mathrm{M}_{\odot}$ and predict the existence of $\sim 3$ unidentified galaxies with $M_{*} \sim 10^{5} \mathrm{M}_{\odot}$ within $300 \mathrm{kpc}$ of the MW. Overall, we find no evidence for the classical missing satellites or too-big-to-fail (TBTF) problems for satellite galaxies in our sample. Among the satellites, TBTF is resolved primarily by subhalo disruption and overall mass-loss; central density profiles of subhaloes are of secondary importance. For non-satellite galaxies, our LG-like simulations predict as many as $\sim 10$ as-ofyet unseen galaxies at distances $0.3-1 \mathrm{Mpc}$ from both hosts, with $M_{*} \simeq 10^{5-6} \mathrm{M}_{\odot}$ (in haloes with $V_{\max } \sim 20 \mathrm{~km} \mathrm{~s}^{-1}$ ), albeit with large halo-to-halo variance. None of our simulations produces a compact, baryon-dominated, high-density dwarf elliptical-type galaxy (with $V_{\text {circ }}$ $\gtrsim 35 \mathrm{~km} \mathrm{~s}^{-1}$ at $r<1 \mathrm{kpc}$ ), of which six may appear in the LG (but none in the MW). It may therefore remain a challenge to reproduce the full diversity of the dwarf population, including both the highest and lowest density systems.
\end{abstract}

Key words: galaxies: dwarf-galaxies: formation-galaxies: Local Group-cosmology: theory.

\section{INTRODUCTION}

Our location within the Local Group (LG) affords it a unique importance in astronomy. It remains the only part of the Universe where we can detect tiny dwarf galaxies (stellar mass $M_{*} \lesssim 10^{6} \mathrm{M}_{\odot}$ ), let alone use resolved stellar observations to study their internal properties

\footnotetext{
^E-mail: sheagk@caltech.edu

$\dagger$ Einstein Fellow.

$\ddagger$ Caltech-Carnegie Fellow.
}

and kinematics. As the most dark matter-dominated galaxies in the Universe (e.g. McConnachie 2012), these dwarf galaxies provide crucial tests of the standard structure formation paradigm, cold dark matter with a cosmological constant [Lambda cold dark matter $(\Lambda \mathrm{CDM})]$, and may ultimately indirectly reveal the nature of DM itself (e.g. Ackermann et al. 2015).

While $\Lambda$ CDM reproduces large-scale observations extraordinarily well (e.g. Springel et al. 2005), explaining the dwarf galaxy population within the $\Lambda \mathrm{CDM}$ framework has historically proven difficult (see Bullock \& Boylan-Kolchin 2017, for a recent review). 
Perhaps most famously, the 'missing satellites' problem (MSP; Klypin et al. 1999; Moore et al. 1999) points out that dark matteronly (DMO) simulations of MW-mass hosts in $\Lambda \mathrm{CDM}$ predict orders of magnitude more bound subhaloes within $\sim 300 \mathrm{kpc}$ than known luminous satellites of the MW. While the MSP is usually accounted for by a combination of photoionization during reionization (Bullock, Kravtsov \& Weinberg 2000; Somerville 2002), observational bias and incompleteness (e.g. Tollerud et al. 2008), and subhalo destruction due to the MW disc (D'Onghia et al. 2010; Garrison-Kimmel et al. 2017; Sawala et al. 2017), these solutions typically resolve the disparity by placing the known MW satellites in the largest subhaloes predicted around MW-mass hosts and leaving the smallest clumps undetected or entirely dark. This picture is further supported by the success of applying extrapolations of the abundance matching paradigm, which successfully reproduces large-scale clustering statistics by assuming a relatively tight relationship between halo mass $M_{\text {halo }}$ and $M_{*}$, to the LG environment (Garrison-Kimmel et al. 2014a).

However, the too-big-to-fail (TBTF) problem notes that the circular velocity profiles of the largest subhaloes in DMO simulations of MW-mass galaxies (i.e. the subhaloes assumed to host the luminous satellites) are incompatible with observational constraints on the MW dwarf satellites (Boylan-Kolchin, Bullock \& Kaplinghat 2011, 2012). A similar discrepancy exists when comparing with the satellite galaxies of M31 (Tollerud, Boylan-Kolchin \& Bullock 2014) or the dwarf galaxies in the Local Field (defined here as within $1 \mathrm{Mpc}$ of the MW or M31, but more than $300 \mathrm{kpc}$ from both; Garrison-Kimmel et al. 2014b), and TBTF even appears to exist beyond the LG entirely (Papastergis et al. 2015; Papastergis \& Shankar 2016): dwarf galaxies $\left(M_{*} \sim 10^{5-7} \mathrm{M}_{\odot}\right)$ have less mass within $\sim(250 \mathrm{pc})-(1 \mathrm{kpc})$ than DMO simulations of the haloes expected to host those galaxies predict.

Recent simulations have begun to jointly resolve the $\mathrm{MSP}^{1}$ and TBTF by more realistically modelling gas cooling, star formation, and stellar/supernovae feedback. For example, Brooks \& Zolotov (2014), using simulations from Zolotov et al. (2012), demonstrated a reduction in the peak circular velocity of the haloes associated with TBTF due to a combination of supernovae feedback (modelled via the 'blastwave' scheme of Stinson et al. 2006) and tidal disruption, such that their simulations were free of both TBTF and the MSP. More recently, Dutton et al. (2016) and Buck et al. (2019) showed that the NIHAO simulation suite, which also adopts the blastwave scheme, is similarly free of the MSP and TBTF.

The conclusion that TBTF and the MSP can be explained via baryonic physics, even using non-blastwave feedback implementations, is growing increasingly robust. The APOSTLE simulations (Fattahi et al. 2016b; Sawala et al. 2016b), for example, apply the EAGLE models for galaxy formation, which are tuned to reproduce the stellar mass function (SMF) and sizes of galaxies at $z=0.1$ (Crain et al. 2015; Schaye et al. 2015), to 12 LG-like volumes, ${ }^{2}$

\footnotetext{
${ }^{1}$ The Auriga simulations (Grand et al. 2017), high-resolution magnetohydrodynamic zoom-ins focusing on isolated MW-mass galaxies, also reproduce the MW/M31 satellite luminosity functions down to $5 \times 10^{5} \mathrm{M}_{\odot}$ (Simpson et al. 2018), though to date there have been no analyses of the internal structure of those satellites.

${ }^{2}$ The APOSTLE simulations follow in the spiritual footsteps of the CLUES (Constrained Local UniversE Simulations) project (e.g. Gottloeber, Hoffman \& Yepes 2010) in targeting LG-like pairs in hydrodynamic, cosmological zoom-in simulations. The CLUES simulations, however, constrain the $\sim 5 h^{-1} \mathrm{Mpc}$ environment around the targeted hosts to match that of the actual LG.
}

demonstrating that extrapolations of models that match the statistics of larger galaxies can also duplicate the LG. The APOSTLE dwarf galaxy populations generally do not exhibit the MSP: the simulated volumes contain a similar number of galaxies with $M_{*} \geq 10^{5} \mathrm{M}_{\odot}$ as the actual MW, M31, and LG. Moreover, the mass function of subhaloes that host the luminous dwarf galaxies in APOSTLE (quantified by $V_{\max }$, the peak of the circular velocity curve) agrees with the mass function implied by the Peñarrubia, McConnachie \& Navarro (2008) estimates for the MW dwarf spheroidals (dSphs), implying that the APOSTLE hosts are also free of the TBTF problem.

In an alternative approach, Wetzel et al. (2016) used the Feedback In Realistic Environments (FIRE; Hopkins et al. 2014, $2017)^{3}$ physics to simulate an isolated MW-mass galaxy with high enough resolution to capture the internal dynamics of the classical satellites. FIRE includes explicit models for star formation and stellar/supernovae feedback that self-consistently yield bursty star formation in dwarf galaxies (Muratov et al. 2015; El-Badry et al. 2016; Sparre et al. 2017; Faucher-Giguère 2018) and overall agreement with a variety of galaxy-scale observables, including the star formation histories of dwarf galaxies (Oñorbe et al. 2015; Wetzel et al. 2016; Fitts et al. 2017); the mass-metallicity (Ma et al. 2016), stellar mass-halo mass (Hopkins et al. 2014, 2018), and stellar mass-star formation rate (Sparre et al. 2017) relationships; and the fraction of the stellar mass in the haloes of MW-mass galaxies (Sanderson et al. 2017). Wetzel et al. (2016) showed that FIRE also yields a reasonable MW satellite population: the set of simulated dwarf galaxies falls roughly midway between that of the MW and M31 when counting galaxies either by $M_{*}$ or by the lineof-sight stellar velocity dispersion $\sigma_{*}$, the observable relevant to TBTF.

These works, however, have suffered from limitations. While the hosts in the APOSTLE simulations are carefully selected to match the LG environment, the majority of the APOSTLE results are drawn from their 'L2' simulations with baryonic particle masses $\sim 10^{5} \mathrm{M}_{\odot}$, approaching the total mass of the smaller classical dwarf galaxies. In addition, the effective equation of state and the spatial/density resolution used in the APOSTLE simulations are such that the smallest resolvable Jeans/Toomre mass is $>10^{8} \mathrm{M}_{\odot}$; therefore, clouds in lower mass galaxies cannot be self-consistently resolved. The simulations in Zolotov et al. (2012) and Buck et al. (2019) similarly have baryonic particle masses $>20000 \mathrm{M}_{\odot}$, with the highest resolutions reached at lower halo masses $\sim 8 \times 10^{11} \mathrm{M}_{\odot}$. Wetzel et al. (2016) reached higher resolutions and used a more physical subgrid model for star formation and feedback, but their results are based on a single simulation of an isolated host, rather than an LG-like environment.

Here we introduce the first in a set of simulations that apply the FIRE physics to LG-like volumes at state-of-the-art resolution. We present two simulated LG-like pairs (containing four MWmass analogues), along with six isolated MW-mass galaxies for comparison. Our simulations generally reproduce the observed properties of dwarf galaxies in the LG: they do not suffer from either the MSP or TBTF when including baryonic physics.

This paper is organized as follows. In Section 2, we describe the simulations and briefly review the star formation and feedback models. Section 3 details our methods for compiling our observed and simulated galaxy catalogues. Section 4 presents the SMFs of our simulated hosts, counting both satellites and non-satellites.

\footnotetext{
${ }^{3}$ http://fire.northwestern.edu
} 
Section 5 then examines the internal structure of our simulated dwarfs by comparing their central masses to those implied by observations via circular velocity curves. Section 6 presents the relationships between stellar kinematics, stellar mass, and halo mass. We summarize our results and conclusions in Section 7.

\section{SIMULATIONS}

We analyse hydrodynamic, cosmological zoom-in (Katz \& White 1993; Oñorbe et al. 2014) simulations, initialized with MUSIC (Hahn \& Abel 2011), from the FIRE project (Hopkins et al. 2014), run using the improved 'FIRE-2' version of the code from Hopkins et al. (2018). All of the simulations were run using GIZMO (Hopkins 2015), ${ }^{4}$ a multimethod gravity plus hydrodynamics code, in meshless finite-mass ('MFM') mode. This is a meshfree Lagrangian finite-volume Godunov method that automatically provides adaptive spatial resolution while maintaining conservation of mass, energy, and momentum (for extensive tests, see Hopkins 2015). Gravity is solved with an improved version of the TREEPM solver from GADGET-3 (Springel 2005), with fully adaptive (and fully conservative) gravitational force softenings for gas (so hydrodynamic and force softenings are always self-consistently matched), following Price \& Monaghan (2007).

The FIRE physics and source code are nearly identical to those in previous FIRE-2 simulations, with the lone exception that all of our simulations additionally include subgrid turbulent metal diffusion, which produces more realistic metallicity distributions in dwarf galaxies (Escala et al. 2018) but does not alter other galaxywide properties (Hopkins 2017; Su et al. 2017). The FIRE physics modules are described in detail in the papers above, but in brief, we treat radiative heating and cooling from $10-10^{10} \mathrm{~K}$, allow for star formation only in gas that is dense $\left(n>1000 \mathrm{~cm}^{-3}\right)$, Jeans unstable, molecular and self-shielding (Krumholz \& Gnedin 2011), and selfgravitating (Hopkins, Narayanan \& Murray 2013). We then include stellar feedback via radiation pressure, Types Ia and II supernovae, metal mass-loss, and photoionization and photoelectric heating, assuming every star particle represents a single stellar population with a Kroupa (2001) initial mass function (IMF).

We focus on two pairs of LG-like hosts, Romeo \& Juliet and Thelma \& Louise, which are visualized at $z=0$ in Fig. 1. We refer to these simulations (and additional ongoing work) as the 'ELVIS on FIRE' set. The Thelma \& Louise volume was first presented as a DMO simulation as part of the original Exploring the Local Volume In Simulations (ELVIS) suite (Garrison-Kimmel et al. 2014a). Both Thelma \& Loui se and Romeo \& Juliet were also presented at lower resolution and without subgrid metal diffusion in Garrison-Kimmel et al. (2018). We also include the results of six simulations targeting isolated MW-mass haloes; all of these galaxies were also analysed in Garrison-Kimmel et al. (2018), but here we present higher resolution resimulations of $\mathrm{m} 12 \mathrm{~b}, \mathrm{~m} 12 \mathrm{C}$, and $\mathrm{m} 12 \mathrm{z}$ that additionally include subgrid metal diffusion. $\mathrm{m} 12 \mathrm{~b}-\mathrm{m} 12 \mathrm{~m}$ are part of the 'Latte Suite', a set of hosts homogeneously selected to be isolated and roughly the same mass as the MW: $M_{200_{\mathrm{m}}}=(1-2) \times$ $10^{12} \mathrm{M}_{\odot} \cdot{ }^{5} \mathrm{~m} 12 \mathrm{i}$, in particular, uses the same initial conditions as the halo presented in Wetzel et al. (2016), originally taken from the

\footnotetext{
${ }^{4}$ http://www.tapir.caltech.edu/ phopkins/Site/GIZMO.html

${ }^{5}$ The Latte haloes were selected based on $M_{200 \mathrm{~m}}$ rather than $M_{\mathrm{vir}}$, so we opt to report that value here. However, we will adopt the Bryan \& Norman (1998) definition of virial mass $M_{\text {vir }}$ elsewhere in this work. For a comparison with other simulations, the mass definitions in our cosmologies and for
}

AGORA project (Kim et al. 2014). The hosts in the Latte Suite were all simulated with identical resolutions: initial baryonic particle masses $m_{\mathrm{b}}=7067 \mathrm{M}_{\odot}$. Because the LG-like pairs were drawn from different box sizes and slightly different cosmologies, ${ }^{6}$ they feature $\sim 2 \times$ better resolutions (Romeo \& Juliet has $m_{\mathrm{b}}=3523 \mathrm{M}_{\odot}$; Thelma \& Louise has $m_{\mathrm{b}}=3990 \mathrm{M}_{\odot}$ ). Finally, m12 z was also chosen from a separate parent box to be slightly lower mass, and is also at slightly higher resolution than the remainder of the isolated sample with $m_{\mathrm{b}}=4174 \mathrm{M}_{\odot}$. All simulations were run with gas softening lengths that are fully adaptive down to $\epsilon_{\min }^{\mathrm{gas}} \simeq 0.5-1 \mathrm{pc}$ and DM force softenings $\simeq 50 \mathrm{pc}$.

The two central galaxies in Romeo \& Juliet are separated by $839 \mathrm{kpc}$, are approaching one another with $v_{\text {rad }}=-93 \mathrm{~km} \mathrm{~s}^{-1}$, and have a tangential velocity of $v_{\tan }=23 \mathrm{~km} \mathrm{~s}^{-1}$. Thelma and Louise are separated by $920 \mathrm{kpc}$, have $v_{\text {rad }}=-107 \mathrm{~km} \mathrm{~s}^{-1}$, and $v_{\tan }=14 \mathrm{~km} \mathrm{~s}^{-1}$. For a comparison, the MW and M31 are separated by $787 \mathrm{kpc}$ (McConnachie et al. 2005) and are approaching one another with $v_{\text {rad }}=-109 \mathrm{~km} \mathrm{~s}^{-1}$ and $v_{\tan }=17 \pm 17 \mathrm{~km} \mathrm{~s}^{-1}$ (van der Marel et al. 2012, though see Salomon et al. 2016 and Carlesi et al. 2016). Both pairs were selected for these high-resolution simulations on the basis of their low tangential velocities and relative lack of (partial) overlap in their Lagrange volumes with other massive haloes outside the LG. We do not constrain or restrict the larger-scale density fields around the LG hosts, i.e. we do not necessarily expect to reproduce the $\sim 5 \mathrm{Mpc}$-scale 'Local Sheet' (McCall 2014). Table 1 presents additional information about the individual hosts, including the distance to the nearest low-resolution particle $r_{\text {contam }}$ and the number of haloes within $1 \mathrm{Mpc}$ excluded from our analysis due to contamination from these particles.

\section{GALAXY CATALOGUES}

In this section, we briefly discuss the observational sources we use for the properties of dwarf galaxies in the LG, along with our method for extracting the equivalent properties for dwarf galaxies from the simulations.

\subsection{Observations}

We build our observational sample primarily off the data compiled in an updated version of the McConnachie (2012) catalogue of local dwarf galaxies. We exclude all 'starred' systems in the catalogue, for which debate remains about their true nature (i.e. galaxy versus globular cluster); the majority of these are much less massive than our resolution. We take stellar mass-to-light ratios from Woo, Courteau \& Dekel (2008) where available, and otherwise assume $M_{*} / L_{\mathrm{V}}=1.6$ (consistent with Martin, de Jong \& Rix 2008b and extrapolations of Bell \& de Jong 2001). We calculate $V_{1 / 2}=$ $V_{\text {circ }}\left(R_{1 / 2}\right)$, the implied circular velocity at the $3 \mathrm{D}$ (deprojected) half-light radius, for the majority of our galaxies with the Wolf et al. (2010) formula, i.e. based on the velocity dispersion of the stars. For the MW dSphs, we use the velocity dispersions presented in Wolf et al. (2010), though we note that Fattahi et al. (2016a) argued

our concentrations are approximately related by $M_{\mathrm{vir}} / M_{200_{\mathrm{c}}} \simeq 1.15$ and $M_{\text {vir }} / M_{200_{\mathrm{m}}} \simeq 0.91$.

${ }^{6}$ All of our simulations assume flat $\Lambda \mathrm{CDM}$ cosmologies with $h=0.68-0.71$, $\Omega_{\mathrm{m}}=0.266-0.31, \Omega_{\mathrm{b}}=0.0455-0.048$, and $\sigma_{8}=0.801-0.82$ (e.g. Larson et al. 2011; Planck Collaboration XIII 2016). These slight differences in cosmology should have a negligible impact on the scale of the LG (e.g. Garrison-Kimmel et al. 2014c). 


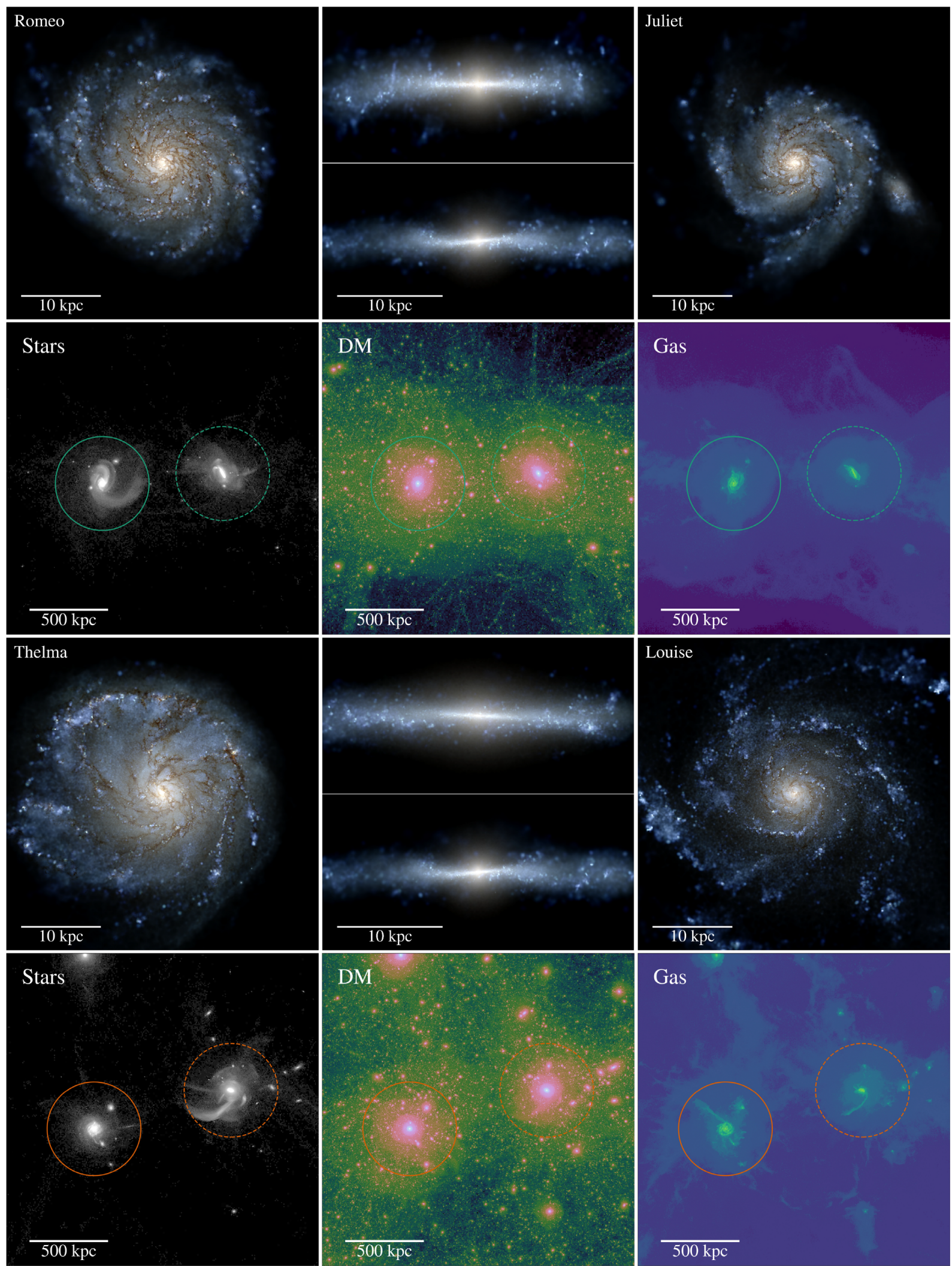

Figure 1. Visualizations of our simulated hosts and their environments. The face-on pseudo-colour images are $40 \mathrm{kpc}$ across; the edge-on images span $30 \mathrm{kpc}$ with a height of $15 \mathrm{kpc}$. The density maps show the highest 3D density along a given line of sight through a cube 2 Mpc on a side, centred on the mid-point of the pair. All of the maps adopt logarithmic colour scales; the stellar maps range from $10^{-9}-3 \times 10^{-2} \mathrm{M}_{\odot} \mathrm{pc}^{-3}$, the dark matter from $10^{-8}-1 \mathrm{M}_{\odot} \mathrm{pc}^{-3}$, and the gas from $10^{-8}-100 \mathrm{M}_{\odot} \mathrm{pc}^{-3}$. The circles around the hosts indicate a radius of $300 \mathrm{kpc}$; the more massive host halo is on the right and is indicated by a dashed circle. The massive galaxy on the outskirts of Thelma \& Louise [with $M_{\mathrm{vir}}=4.5 \times 10^{11} \mathrm{M}_{\odot}, M_{*}(<20 \mathrm{kpc})=1.58 \times 10^{10} \mathrm{M}_{\odot}$ ] is $>1 \mathrm{Mpc}$ from both hosts, excluding it from the analyses that follow. 
Table 1. Basic properties of our host haloes: virial mass $\left(M_{\mathrm{vir}}\right.$, using the Bryan \& Norman 1998 definition), stellar mass within $20 \mathrm{kpc}$ of the central galaxy $\left(M_{*}\right)$, distance to the nearest low-resolution particle $\left(r_{\text {contam }}\right)$, the number of haloes within $1 \mathrm{Mpc}$ with $V_{\max } \geq 10 \mathrm{~km} \mathrm{~s}^{-1}$ that are excluded due to contamination from low-resolution particles $\left(N_{\text {contam }}\right)$, the total mass in the field $[r=(300 \mathrm{kpc})-(1 \mathrm{Mpc})]$ around each host $\left(M_{\mathrm{tot}}^{\text {field }}\right)$, and the number of massive failures identified when comparing subhaloes in the corresponding DMO simulations with the MW dSphs (unaccounted-for subhaloes with $V_{\max }=25-40 \mathrm{~km} \mathrm{~s}^{-1}$; see Section 5 for details); 'strong' massive failures are given in parentheses. We caution that estimates for the virial masses of the MW and M31 frequently vary at the factor of $\gtrsim 2$ level (e.g. Kafle et al. 2018; Patel et al. 2018). Though we do not list it, the total fractional mass contamination within $1 \mathrm{Mpc}$ by low-resolution particles is, at worst, $3.6 \times 10^{-4}$ around m12i. Initial baryonic particle masses are $3523 \mathrm{M}_{\odot}$ in Romeo \& Juliet, $3990 \mathrm{M}_{\odot}$ in Thelma \& Louise, $4174 \mathrm{M}_{\odot}$ in $\mathrm{ml2z}$, and $7067 \mathrm{M}_{\odot}$ in the remaining simulations.

\begin{tabular}{lcccccc}
\hline Host & $\begin{array}{c}M_{\text {vir }} \\
\left(10^{12} \mathrm{M}_{\odot}\right)\end{array}$ & $\begin{array}{c}M_{*} \\
\left(10^{10} \mathrm{M}_{\odot}\right)\end{array}$ & $\begin{array}{c}r_{\text {contam }} \\
(\mathrm{kpc})\end{array}$ & $\begin{array}{c}N_{\text {contam }} \\
(<1 \mathrm{Mpc})\end{array}$ & $\begin{array}{c}M_{\text {tot }}^{\text {field }} \\
\left(10^{12} \mathrm{M}_{\odot}\right)\end{array}$ & $\begin{array}{c}N_{\mathrm{MF}}, \mathrm{MW} \\
(\mathrm{DMO})\end{array}$ \\
\hline M31 & $1.7 \pm 0.3^{a}$ & $\begin{array}{c}\text { Paired hosts } \\
10.3_{-1.7}^{+2.3 b}\end{array}$ & - & - & - & - \\
MW & $1.3 \pm 0.3^{c}$ & $5 \pm 1^{c}$ & - & - & - & - \\
Romeo & 1.24 & 7.37 & 514 & 4 & 0.94 & $10(7)$ \\
Juliet & 1.01 & 4.22 & 1196 & 0 & 0.66 & $15(8)$ \\
Thelma & 1.32 & 7.92 & 1215 & 0 & 1114 & $10(6)$ \\
Louise & 1.03 & 2.86 & 894 & 0 & 0.81 & $8(4)$ \\
m12b & 1.31 & 9.42 & 728 & 0 & 0.64 & $9(6)$ \\
m12C & 1.26 & 6.44 & 1247 & 0 & 1.0 & $14(2)$ \\
m12f & 1.54 & 8.79 & 1110 & 0 & 0.72 & $8(5)$ \\
m12i & 1.07 & 7.00 & 542 & 6 & 0.55 & $13(9)$ \\
m12m & 1.45 & 12.62 & 671 & 3 & 0.82 & $15(9)$ \\
m12 z & 0.80 & 2.24 & 445 & 4 & 0.81 & $7(5)$ \\
\hline
\end{tabular}

Notes: ${ }^{a}$ Diaz et al. (2014);

${ }^{b}$ Sick et al. (2015);

${ }^{c}$ Bland-Hawthorn \& Gerhard (2016).

that work underestimated the uncertainties on the dynamical mass; increasing those uncertainties would tend to reduce the severity of the TBTF problem discussed in Section 5. For the satellites of M31, we take $R_{1 / 2}$ and $V_{1 / 2}$ from Tollerud et al. (2014). The majority of these are based on stellar velocity dispersions, but there are a few exceptions. Most notably, the constraint on M33 only represents the mass of the dark matter halo, taken from a fit to $\mathrm{CO}$ and H I observations (Simon et al. 2006, using data from Corbelli \& Salucci 2000 and Corbelli 2003); including the baryonic component roughly doubles $V_{1 / 2}$. We adopt the total mass estimates (i.e. including baryons) for the remaining M31 satellites, including those that are baryon dominated within $R_{1 / 2}$. For NGC 185 and NGC 147, these are based upon the dynamical modelling of Geha et al. (2010), while the constraint on IC 10 is derived from $\mathrm{HI}$ observations (Wilcots \& Miller 1998). Finally, for the Local Field, we adopt the values $\left(R_{1 / 2}, V_{1 / 2}\right.$, and $\left.\sigma_{*}\right)$ calculated or compiled in Kirby et al. (2014) where possible, though we adopt the modified $V_{1 / 2}$ values presented in Garrison-Kimmel et al. (2014b) for the three galaxies that display evidence of rotation: for the dwarf galaxy WLM, we use the result calculated in detail by Leaman et al. (2012), while we use the method of Weiner et al. (2006, and also see Kirby et al. 2014) to incorporate rotational support into our estimates for Pegasus and Tucana. For all other systems, we fall back on the measurements in McConnachie (2012). We list the properties of the full sample in Appendix A.

\subsection{Simulations}

Because publicly available halo finders are typically tuned to capture DM (sub)haloes, we find unsatisfactory performance when attempting to capture the much more compact stellar clumps (particularly when those clumps are embedded within the stellar halo of a larger host; see Fig. 1). We therefore compile our simulated galaxy catalogues via a multistep process. We first identify bound DM haloes by running AHF (Knollmann \& Knebe 2011) only on the DM particles. We then assign star particles in a first pass to DM clumps via a generous cut on stellar positions and velocities along the direction of motion of the (sub)halo. In a second pass, stars are iteratively removed based on their velocities relative to the velocity dispersion of the system until the latter stabilizes. We then examine each galaxy by hand and repeat the final step with a smaller maximum radius if necessary. Finally, we iteratively compute stellar velocity dispersions independently along the $x$-, $y$-, and $z$-axes, eliminating stars offset by more than $5 \sigma$ from the mean until the dispersion along each axis changes by less than 2.5 per cent; this step typically alters particle counts at the per cent level. However, this step is important for velocity dispersions because contamination by even a single background halo star, with high relative velocity to the satellite, can significantly bias properties such as the radius or velocity dispersion of the satellites. We define $M_{*}$ as the sum of the masses of all the star particles that remain assigned to each galaxy in this way and $\sigma_{*}$ as the RMS average of the $x, y$, and $z$, velocity dispersions of those particles (calculated via the interquartile spacing). Finally, we recompute $V_{\max }$ and $R_{\max }$, the radius at which $V_{\max }$ occurs, using all particles around each host; this step is unimportant for low-mass galaxies, but matters in the higher stellar mass dwarfs where the star particles are a non-negligible fraction of the mass within $R_{\max } \cdot{ }^{7}$ We compute all properties and profiles relative to a halo/galaxy centre defined using a 'shrinkingspheres' approach on the stars (Power et al. 2003). Though there is no explicit requirement at any step that star particles assigned to a given galaxy be bound to the associated halo, our final velocity distributions suggest this is typically the case.

Our approach is similar to Wetzel et al. (2016), but we base our galaxy catalogues on AHF halo catalogues (rather than rockstar; Behroozi, Wechsler \& Wu 2013) and the cuts placed on stellar particles vary slightly; most notably, Wetzel et al. (2016) did not include either our initial cut based on the motion along the direction of the subhalo or our final cut while computing velocity dispersions. Moreover, we quote total line-of-sight velocity dispersions, whereas Wetzel et al. (2016) computed total velocity dispersions at the half-mass radius. Our results are similar: for example, we find an identical number of galaxies with $M_{*} \geq 10^{5} \mathrm{M}_{\odot}$ when applying our method to m12 $i$ as Wetzel et al. (2016) identify in the same halo (simulated without metal diffusion).

In the figures that follow, we plot SMFs down to $M_{*}=$ $7 \times 10^{4} \mathrm{M}_{\odot}$, corresponding to approximately 10 star particles in the lower resolution Latte simulations. While the existence and stellar masses of galaxies above this cut are robust, the internal properties, such as density or velocity dispersion, are more sensitive to resolution and may change with higher resolution simulations (Hopkins et al. 2018). We therefore adopt a slightly higher cut,

\footnotetext{
${ }^{7}$ In cases where the circular velocity curve has no peak/turnover, we instead adopt the inflection point of the curve, i.e. the radius/circular velocity where the curve becomes convex due to the contribution from a background host halo, as $R_{\max }$ and $V_{\max }$.
} 

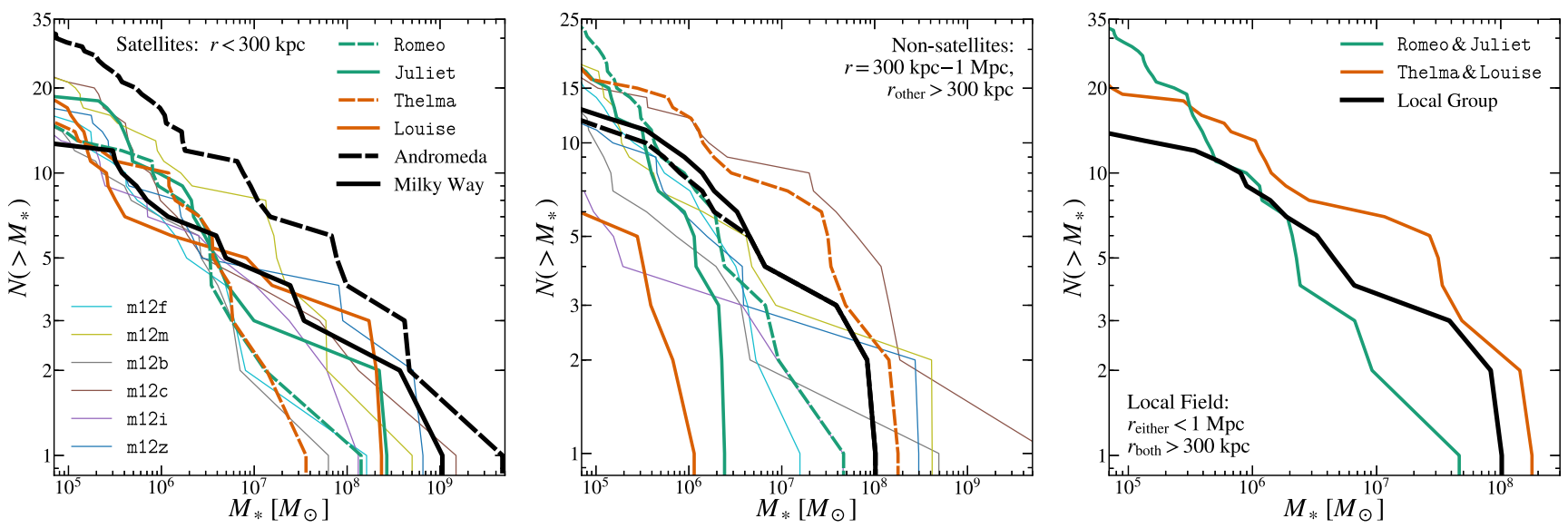

Figure 2. Galaxy SMFs. The panels indicate the satellite population (left; host distance $r_{\text {host }}<300 \mathrm{kpc}$ ), the non-satellite population around each host (centre; $r_{\text {host }}=300-1000 \mathrm{kpc}$, and distance to the paired host $r_{\mathrm{other}}>300 \mathrm{kpc}$ where applicable), and (right) the Local Field (distance from either host $r_{\mathrm{either}}<$ $1 \mathrm{Mpc}$ but distance from both hosts $r_{\mathrm{both}}>300 \mathrm{kpc}$ ). The thin lines indicate the isolated m12 sample, which are sorted in the legend by host virial mass. The satellite SMFs are broadly consistent with that of the MW and M31, though even our richest satellite population slightly (by a factor of $\sim 1.2$ at $10^{5} \mathrm{M}_{\odot}$ ) underproduces that of $\mathrm{M} 31$, possibly because our highest mass host is only $1.45 \times 10^{12} \mathrm{M}_{\odot}$. Similarly, the non-satellite populations around each host are in reasonable agreement with that of the MW and M31, with considerable scatter. The simulated Local Field populations are also generally consistent with observations, particularly for $M_{*} \gtrsim 5 \times 10^{5} \mathrm{M}_{\odot}$; below that, Romeo \& Juliet displays a steep upturn relative to the LG. Thelma \& Loui se, meanwhile, slightly overproduces the Local Field SMF at all masses. We predict a median of 2.5 additional (i.e. undetected) non-satellite galaxies with $M_{*} \geq 10^{5} \mathrm{M}_{\odot}$ and $r_{\mathrm{MW}}=300-1000 \mathrm{kpc}$, along with 4 additional MW satellites with $M_{*}=10^{5}-3 \times 10^{5} \mathrm{M}_{\odot}$.

$M_{*}=10^{5} \mathrm{M}_{\odot}$, corresponding to 14-29 star particles, when quoting galaxy counts or investigating internal structure.

\section{STELLAR MASS FUNCTIONS}

Fig. 2 presents the SMFs of dwarf galaxies throughout the Local Volume. As expected from Garrison-Kimmel et al. (2014a), the satellite SMFs (host distance $r_{\text {host }}<300 \mathrm{kpc}$ ) of the isolated and paired haloes overlap well. Our 10 hosts contain between 12 and 20 satellites with $M_{*} \geq 10^{5} \mathrm{M}_{\odot}$, with a 66 percent scatter of 6.1 galaxies. For a comparison, the scatter in the number of subhaloes around the DMO ELVIS hosts (Garrison-Kimmel et al. 2014a) above an equivalent peak halo mass (using the zero-scatter stellar mass versus peak halo mass relationship from that work) is 20.5. However, the host masses from ELVIS also vary more widely than the sample presented here: the DMO ELVIS host masses have a 66 percent scatter of $1.25 \times 10^{12} \mathrm{M}_{\odot}$, while that of our sample is only $0.37 \times 10^{12} \mathrm{M}_{\odot}$. Naively scaling the two values by one another [i.e. scatter in $N_{\text {sats }}\left(\mathrm{M} \geq 10^{5} \mathrm{M}_{\odot}\right) /$ scatter in host $M_{\text {vir }}$ ] yields nearly identical values, such that our results are consistent with the FIRE simulations predicting the same degree of scatter in the number of luminous satellites as DMO simulations.

The FIRE satellite populations also provide a good match to the MW satellite SMF, particularly below the masses of the LMC and $\mathrm{SMC},{ }^{8}$ though the agreement is not perfect: the simulated galaxies host a median of 15.5 satellites with $M_{*} \geq 10^{5} \mathrm{M}_{\odot}$, compared with the 12 such known MW satellites, and we typically predict an SMF that continues to rise between the relatively bright classical dSphs $\left(M_{*} \gtrsim 3 \times 10^{5} \mathrm{M}_{\odot}\right)$ and the ultra-faints dwarfs $\left(M_{*} \lesssim 3 \times 10^{4} \mathrm{M}_{\odot}\right)$ identified in deep surveys such as SEGUE

\footnotetext{
${ }^{8}$ The worse agreement at the high-mass end is not particularly unexpected: none of our hosts were selected to contain an LMC-mass satellite, and a randomly selected MW/M31-mass halo is statistically unlikely to have LMC- or M33-mass satellites (Busha et al. 2011; Tollerud et al. 2011).
}

(Belokurov et al. 2009) and DES (Drlica-Wagner et al. 2015). The difference is small relative to the order-of-magnitude difference referred to by the MSP - we predict a median of 4 satellites with $M_{*}=10^{5}-3 \times 10^{5} \mathrm{M}_{\odot}-$ but it may suggest additional, relatively luminous, undetected satellites (also see Tollerud et al. 2008). Rather than a sign of observational incompleteness, the flattening of the MW SMF may instead reflect a feature from reionization (see Bose, Deason \& Frenk 2018); if so, our simulations do not capture such a feature overall.

In contrast to the relative agreement with the MW SMF, all of the simulated satellite SMFs lie slightly below that of M31. Our hosts have, on average, 54 per cent as many satellites with $M_{*} \geq 10^{5} \mathrm{M}_{\odot}$ as are already known around M31. The offset in the mean counts relative to M31 is roughly constant for $M_{*} \lesssim 10^{7} \mathrm{M}_{\odot}$ (at which point the mean difference becomes even larger), indicating that M31 contains systematically more satellites at fixed stellar mass than our simulated hosts. For a comparison, the mean offset between the simulated satellite populations and that of the MW is $\sim 2$ per cent at the mass of $\mathrm{CVnI}\left(3 \times 10^{5} \mathrm{M}_{\odot}\right)$ and remains under 20 per cent over two orders of magnitude (up to the mass of Fornax, $2.4 \times 10^{7} \mathrm{M}_{\odot}$ ). The difference in satellite counts is clear, but not extreme: our host with the largest number of satellites (m12m, with $M_{\text {vir }}=1.45 \times$ $10^{12} \mathrm{M}_{\odot}$ ) contains 73 per cent as many galaxies above $10^{5} \mathrm{M}_{\odot}$ with an average of 74 per cent from $10^{5}$ to $3 \times 10^{7}$. As we show in Appendix B, this result is only marginally sensitive to the radial cut used to separate satellites from non-satellites. It is also qualitatively independent of the assumed mass-to-light ratio for the observed dwarf galaxies: even adopting a stellar mass-to-light ratio of unity for the galaxies not included in Woo et al. (2008) yields a mean of 61 per cent as many satellites as M31 with $M_{*}=10^{5} \mathrm{M}_{\odot}$.

In collisionless simulations, the subhalo mass function scales closely with the host halo mass (e.g. Boylan-Kolchin et al. 2010). It is therefore natural to expect that the specific predictions in Fig. 2 depend on the halo masses of our hosts. If so, then the abundance of dwarf galaxies around M31 (relative both to the MW and to our simulated hosts) may point towards a higher 


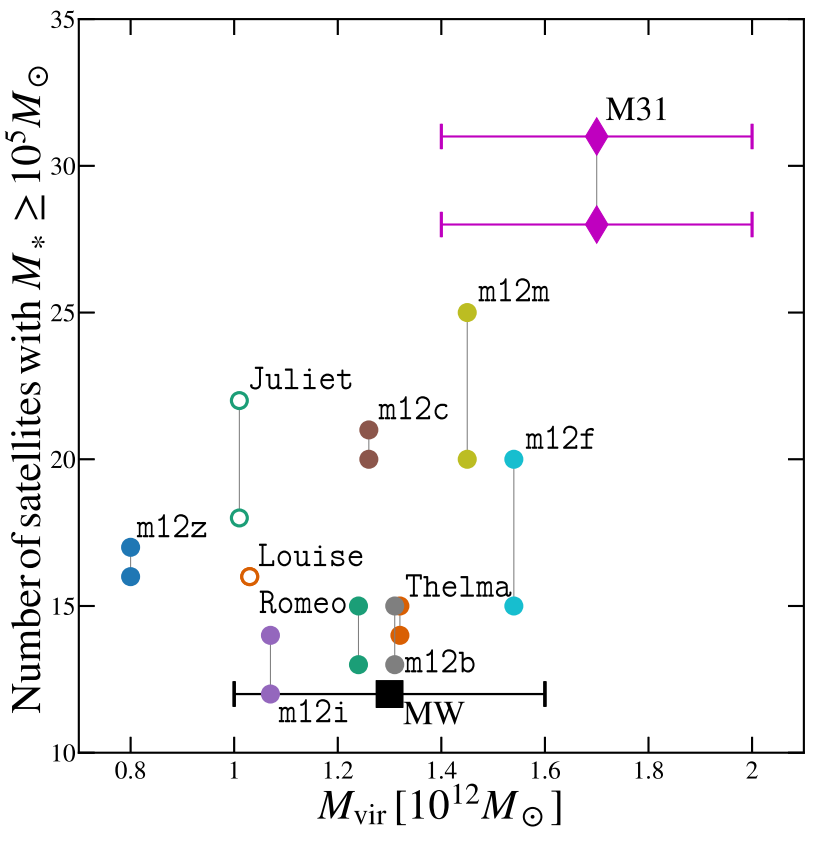

Figure 3. The number of dwarf galaxies with $M_{*} \geq 10^{5} \mathrm{M}_{\odot}$ within $300 \mathrm{kpc}$ (lower points) and $400 \mathrm{kpc}$ (upper points) of each host, as a function of host virial mass. Colours are identical to Fig. 2, with the lower mass host in the LG-like pairs plotted as open points. Counts around M31 and the MW are also plotted, with mass estimates taken from Diaz et al. (2014) and BlandHawthorn \& Gerhard (2016), respectively. Both the MW and Loui se have zero satellites with $M_{*} \geq 10^{5} \mathrm{M}_{\odot}$ between 300 and $400 \mathrm{kpc}$ (Samuel et al., in preparation), and therefore have only a single value plotted.

M31 halo mass. Large-scale estimates for the mass of M31 typically suggest $M_{\mathrm{vir}, \mathrm{M} 31} \gtrsim 1.5 \times 10^{12} \mathrm{M}_{\odot}$; for example, Diaz et al. (2014) used the net momentum of the LG to estimate $M_{\mathrm{vir}, \mathrm{M} 31}=1.7 \pm 0.3 \times 10^{12} \mathrm{M}_{\odot}$. However, Kafle et al. (2018) recently argued for $M_{\mathrm{vir}, \mathrm{M} 31}=0.8 \pm 0.1 \times 10^{12} \mathrm{M}_{\odot}$ by applying a Bayesian framework to high-velocity planetary nebulae. Fig. 3 shows the number of dwarf galaxies near each host, as a function of host virial mass. Though the trends with mass are weak (e.g. our lowest mass host contains the fifth most satellites), our results suggest that it is difficult to match both the SMF of the MW and of M31 without a higher virial mass for M31. The weak trend may be due simply to a large amount of dwarf-to-dwarf scatter in galaxy formation efficiency, or it could be because smaller host haloes tend to have lower mass centrals, resulting in less subhalo destruction and washing out the correlation between subhalo abundance and host $M_{\text {halo }}$ (Garrison-Kimmel et al. 2017). Using a similar sample of simulations, Samuels et al. (2019) find that the number of satellites within 50 and $100 \mathrm{kpc}$ declines particularly strongly with host $M_{*}$, lending support to the latter possibility. If the latter is the case, then the lack of a trend in Fig. 3 must be read carefully, as the galaxy mass varies along with the halo mass in that figure. Given that the mass of the MW galaxy is fixed, varying the assumed MW halo mass should result in corresponding changes in the predicted SMF.

Broadly speaking, the non-satellite SMFs in Fig. $2\left(r_{\text {host }}=300\right.$ $1000 \mathrm{kpc}$, and excluding satellites of the paired host if applicable) generally agree with counts in the fields around the MW/M31. However, there are again hints of undetected galaxies with $M_{*} \gtrsim$ $10^{5} \mathrm{M}_{\odot}$ : we predict a median of 14.5 galaxies with $M_{*} \geq 10^{5} \mathrm{M}_{\odot}$, compared to the 12 known around the MW. Furthermore, increasing the mass of our M31 analogue may result in even more predicted dwarfs; our predictions in the Local Field may be a lower limit. If ultra-diffuse galaxies (UDGs) are prevalent in the field (as predicted by Di Cintio et al. 2017 and Chan et al. 2017), with central surface brightnesses 24-26 mag $\operatorname{arcsec}^{-2}$ (van Dokkum et al. 2015), then some of this incompleteness may even arise at $M_{*} \sim 10^{7} \mathrm{M}_{\odot}$. Surprisingly (as Garrison-Kimmel et al. 2014a predict 75 per cent more haloes above fixed $V_{\max }$ in DMO halo counts), there is no clear offset in the Local Field SMFs between the isolated and paired hosts, though all of the latter except Louise are on the upper edge of the distribution. However, our statistics remain relatively small, and we require a larger, mass-selected sample to make strong statements regarding the efficiency of galaxy formation in dwarfs within $\sim 1 \mathrm{Mpc}$ of an LG-like pair versus an isolated MW-mass galaxy. We caution that the lines representing Romeo and Juliet (Thelma and Louise) are not completely independent, with the volumes probed overlapping by 42 per cent (37 per cent).

Finally, the right-hand panel of Fig. 2 plots the SMF of the 'Local Field' (all non-satellite galaxies within $1 \mathrm{Mpc}$ of either of the hosts). The observed Local Field SMF lies roughly in between our two simulated LGs for $M_{*} \gtrsim 5 \times 10^{5} \mathrm{M}_{\odot}$. Consistent with the centre panel, some amount of observational incompleteness is possible, and perhaps even likely, but more simulations are required: both the real Local Field and Thelma \& Louise contain 5 nonsatellite galaxies with $M_{*}=10^{5}-10^{6} \mathrm{M}_{\odot}$, while Romeo \& Juliet contains 19. Thelma \& Louise, however, does overproduce the observed SMF at all masses, predicting a total of 18 galaxies with $M_{*}>10^{5} \mathrm{M}_{\odot}$ compared to the only 13 known in the LG. The comparison with the fields around our larger sample of isolated hosts clearly demonstrates very large systematic halo-to-halo variations in this prediction though. The number of galaxies in the Local Field may also scale with the total mass in that volume, which is extremely difficult to accurately constrain. However, the Local Field around Romeo \& Juliet contains $\sim 80$ per cent as much mass as that around Thelma $\&$ Louise, ${ }^{9}$ but $\sim 10$ more galaxies with $M_{*} \geq 10^{5} \mathrm{M}_{\odot}$.

In Appendix B we consider the effects of a slightly larger $(\sim 400 \mathrm{kpc}$ ) radial cut used to assign satellites their hosts, and show that this does not qualitatively alter our conclusions above. However, it somewhat decreases the tension with both M31 and the Local Field by reassigning a few galaxies from the field to the M31 analogue.

\section{TВ TF}

Due to the resolution required to study the inner $\sim 500 \mathrm{pc}$ of simulated dwarf subhaloes, TBTF was originally defined using DMO simulations. Boylan-Kolchin et al. (2011) therefore focused on the $\mathrm{dSph}$ satellites of the MW. Because dSphs are dispersion supported, a measurement of $\sigma_{*}$ provides a robust estimate of $V_{1 / 2}$. Moreover, the high dynamical mass-to-light ratios implied by $\sigma_{*}$ suggest that $\mathrm{dSphs}$ are strongly DM-dominated, indicating that the estimates on $V_{1 / 2}$ may be fairly compared to the subhalo masses provided by DMO simulations. Later work on TBTF that expanded beyond the MW satellites (e.g. Garrison-Kimmel et al. 2014b; Tollerud et al. 2014) typically sought to recast observational measurements for non-dispersion supported systems into similar constraints on $V_{1 / 2}$, and either excluded or treated separately galaxies with significant baryonic mass within $R_{1 / 2}$ (for which $V_{1 / 2}$ is not fairly comparable to the results of DMO simulations).

\footnotetext{
${ }^{9}$ The former (Romeo \& Juliet) has a total $1.2 \times 10^{12} \mathrm{M}_{\odot}$, while the latter contains $1.5 \times 10^{12} \mathrm{M}_{\odot}$.
} 
Approaches to TBTF using baryonic simulations have varied. For example, Sawala et al. (2016b) showed that the number of luminous subhaloes in the APOSTLE simulations above a given $V_{\max }$ agrees with estimates for the MW satellite population from Peñarrubia et al. (2008). They then obtain separate $V_{\max }$ estimates for the MW satellites by matching them with dwarf galaxies in their simulations based on $M_{*}, V_{1 / 2}$, and $R_{1 / 2}$; the $V_{\max }-M_{*}$ relationship implied by these estimates is in good agreement with the simulated relationship. Wetzel et al. (2016), conversely, sought to compare directly with the data: they showed good agreement between the dwarf satellites of $\mathrm{m} 12 i$ and those of the MW/M31 when counting galaxies by stellar velocity dispersion and when viewed in velocity dispersion - stellar mass space.

Here, we adopt a hybrid approach. We first demonstrate that the DMO simulations of our host haloes suffer from TBTF by reproducing the Garrison-Kimmel et al. (2014b) analysis on the DMO simulations, then show that the same analysis applied to the luminous dwarf galaxies in the FIRE simulations yields no such discrepancy. Because direct comparisons with data are ideal, we will demonstrate in Section 6 that the simulated dwarfs also broadly reproduce the observed relationship between stellar mass and stellar velocity dispersion. However, because we will compare our simulated dwarfs to non-satellite galaxies and to more massive systems, for which the assumption of dispersion-dominated kinematics is not well-motivated, we begin by inspecting the central masses of our simulated systems and their observational counterparts.

We therefore begin by generally replicating the analyses of Boylan-Kolchin et al. (2012), Tollerud et al. (2012), and GarrisonKimmel et al. (2014b), who identified problematic (sub)haloes by comparing the circular velocity curves of simulated systems with constraints on observed dwarf galaxies. Before presenting the results of this analysis, we first describe our methods for calculating the rotation curves in the DMO and hydrodynamic simulations, then briefly review the galaxies included on each plot, and finally summarize our nomenclature and methods for identifying and counting the problematic (sub)haloes.

\subsection{Methods}

\subsubsection{Calculating circular velocity curves}

For the DMO simulations, we follow previous TBTF analyses in computing circular velocity curves for the (sub)haloes by normalizing a fixed density profile to the large-scale properties of each system. We assume NFW (Navarro, Frenk \& White 1996) profiles for the DMO systems, scaled to $R_{\max }$ and $V_{\max }$ of each halo, but, as we discuss in Section 5.4, this has a second-order effect on our conclusions: adopting the raw particle data from the DMO simulations (and ignoring the impact of gravitational softening) does not alter our conclusions.

Meanwhile, for the hydrodynamic simulations, we follow Boylan-Kolchin et al. (2012) in fitting density profiles (here taken to be the $\alpha, \beta, \gamma$ model; e.g. Jaffe 1983; Hernquist 1990; Merritt et al. 2006 or Di Cintio et al. 2014b) to the resolved portion of each halo, then extrapolating the fits inward to compute $V_{\text {circ }}(r)$. Based on section 4.1.4 of Hopkins et al. (2018), who argued that the usual Power et al. (2003) relaxation time criterion is equivalent to a limit on the number of enclosed particles, we take $r_{\min }$ [the minimum radius used in fitting the density and the radius within which we adopt the extrapolated $M(r)$ ] as the radius containing 300 DM particles; we adopt an outer radius for the fit of $15 \mathrm{kpc}$. Appendix C directly examines the (minimal) impact of varying $r_{\text {min }}$, and compares $V_{\text {circ }}$ from the extrapolated fits to the raw data and to NFW profiles. Importantly, as with the DMO simulations, we show in Section 5.4 that the shape of the central profile has only a marginal impact on the number of massive failures that we identify in the hydrodynamic simulations, even among non-satellite galaxies.

\subsubsection{Selecting galaxies and haloes}

We separately analyse satellites of the MW, satellites of M31, and galaxies in the Local Field, where satellites are again defined as galaxies within $300 \mathrm{kpc}$ of each host. We include every galaxy that meets each distance cut and has velocity information that is representative of the mass of the galaxy. This breaks slightly from the analyses of Boylan-Kolchin et al. $(2011,2012)$ and GarrisonKimmel et al. (2014b), who eliminated the LMC, the SMC, and NGC 6822 for various reasons. In contrast, we eliminate only the Sagittarius dSph. Because Sagittarius is in the process of tidally disrupting, stellar kinematics do not necessarily probe the underlying dynamical mass. Consequently, we may identify a single subhalo as a 'massive failure' (defined in detail in Section 5.1.3) that could be associated with Sagittarius, increasing our counts below by one. We generally adopt the constraints at $\left(R_{1 / 2}, V_{1 / 2}\right)$ detailed in Section 3.1, but the wealth of data on the Magellanic Clouds allows us to plot rotation curves for those systems. Specifically, we adopt the H Ibased rotation curve for the SMC from Stanimirović, StaveleySmith \& Jones (2004) and the proper motion-based rotation curve for the LMC from van der Marel \& Kallivayalil (2014). Finally, we note that M32 lies outside the limits of the central panel (in the upper left, at $R_{1 / 2}=110 \mathrm{pc}, V_{1 / 2}=79 \mathrm{~km} \mathrm{~s}^{-1}$ ), and Leo T lies outside the limits of the right-hand panel (at $R_{1 / 2}=152 \mathrm{pc}, V_{1 / 2}=$ $13 \mathrm{~km} \mathrm{~s}^{-1}$ ). Though these points are not shown on the axes, they are included when identifying massive failures.

For the DMO simulations, we seek to reproduce the cuts adopted by previous TBTF analyses. However, because we lack evolutionary histories for our (sub)haloes, we select on present-day $V_{\max }$ instead of adopting the $\max \left[V_{\max }(t)\right]>30 \mathrm{~km} \mathrm{~s}^{-1}$ cut used in, e.g. GarrisonKimmel et al. (2014b). Based on fig. 1 of Boylan-Kolchin et al. (2012) and the results of Garrison-Kimmel et al. (2014b), we consider (sub)haloes with $V_{\max } \geq 25 \mathrm{~km} \mathrm{~s}^{-1}$. For satellites, this cut is typically more conservative than the criteria of Garrison-Kimmel et al. (2014b) as many subhaloes that reached $V_{\max } \gg 30 \mathrm{~km} \mathrm{~s}^{-1}$ can be stripped to $V_{\max } \leq 20 \mathrm{~km} \mathrm{~s}^{-1}$ today (e.g. Sawala et al. 2016a). In principle, however, we may include some systems (particularly in the Local Field) that only recently reached their present-day mass, and which may therefore be expected to remain 'dark' (e.g. Fitts et al. 2017). However, as we will show below, there are enough systems with $V_{\max } \geq 25 \mathrm{~km} \mathrm{~s}^{-1}$ in the field that this is unlikely to change our conclusions. ${ }^{10}$

For the hydrodynamic simulations, we opt to reproduce the cuts placed on the observed galaxies. That is, we select galaxies based on $M_{*}$, rather than $V_{\max }{ }^{11}$ We select all luminous galaxies with

\footnotetext{
${ }^{10}$ Our results with respect to the DMO simulations are insensitive to these cuts. For example, we find qualitatively identical results if we select potential massive failures by their circular velocity at fixed radius, rather than by $V_{\max }$. Specifically, selecting the 12 subhaloes with the largest circular velocities at $r=1 \mathrm{kpc}$, rather than all subhaloes with $V_{\max } \geq 25 \mathrm{~km} \mathrm{~s}^{-1}$, still yields at least one, and typically $\gtrsim 3$, satellite with $V_{\text {circ }}$ profiles that are incompatible with all of the MW dSphs (i.e. massive failures).

${ }^{11}$ Note, however, that we do assign galaxies to host the LMC and SMC based on their $V_{\max }$, rather than $M_{*}$, which is a more stringent cut (see Fig. 6).
} 
$M_{*} \geq 10^{5} \mathrm{M}_{\odot}$. As we show explicitly in Section 6, this cut is less restrictive than a $V_{\max }$-based cut: it includes many haloes with $V_{\max }$ $\ll 25 \mathrm{~km} \mathrm{~s}^{-1}$, and only excludes three with $V_{\max } \geq 25 \mathrm{~km} \mathrm{~s}^{-1}$. Based on Fig. 2, this is a conservative estimate for a stellar mass-based cut: the simulations all match or slightly exceed the MW SMF at $10^{5} \mathrm{M}_{\odot}$. The same is true in the Local Field: while observational completeness in the Local Field is poorly defined, Fig. 2 shows that there are likely undetected galaxies at $M_{*} \lesssim 5 \times 10^{5} \mathrm{M}_{\odot}$.

\subsubsection{Identifying massive failures}

We adopt the nomenclature of Garrison-Kimmel et al. (2014b) in defining 'strong massive failures' and 'massive failures' separately. Around the MW, the former are subhaloes that are too dense to host any of the MW dSphs, while the latter have rotation curves consistent with either Draco or Ursa Minor (or both), but cannot be associated with those galaxies because they have already been assigned to other subhaloes. In other words, strong massive failures have circular velocity curves that lie above all of the MW dSphs, while massive failures are 'leftover' systems that are otherwise consistent with either Draco or Ursa Minor, but that are kinematically incompatible with the remainder of the MW dSphs.

Due to the wide variability in the internal structures of dwarfs around M31 and in the Local Field, we opt to apply the same nomenclature to those volumes but insist that every galaxy be associated with a single halo (rather than just Draco and Ursa Minor). In practice, we therefore identify massive, unaccountedfor haloes. As demonstrated by Garrison-Kimmel et al. (2014b), applying a stellar mass versus halo mass relationship that reproduces counts in the LG (when applied to DMO simulations) to these unaccounted-for haloes assigns them $M_{*} \geq 5 \times 10^{5} \mathrm{M}_{\odot}$. Therefore, the massive failures we identify around M31 and in the Local Field would be nominally expected to host bright galaxies.

\subsection{Results: DMO simulations}

Fig. 4 presents the results of performing these analyses on the DMO simulations. We compare the satellites of the lower (higher) mass host in each pair to those of the MW (M31) in the left-hand (central) panel, and show the Local Field population in the right-hand panel. Strong massive failures (which only exist in comparison with the MW satellites) are plotted as black lines, while massive failures are indicated by the dashed grey lines. These latter sets are massive, dense (sub)haloes that we nominally expect to form stars, yet that lack an observational counterpart. Haloes assigned to host a galaxy (which are not counted as massive failures) are indicated by the magenta lines. Juliet contains analogues for both the LMC and SMC; these subhaloes are indicated by the long and short dashed magenta lines, respectively.

As expected, we identify several (strong) massive failures in the left-hand panel. However, our analysis identifies only one massive failure when comparing Romeo to the M31 satellite population, and none among the satellites of Thelma, though our analysis places several galaxies in subhaloes that are likely not massive enough to host them. As a glaring example, none of the satellites of The lma have $V_{\max } \geq 50 \mathrm{~km} \mathrm{~s}^{-1}$, but four are assigned to host M33, M32, NGC 205, and NGC 147, all of which have $M_{*} \gtrsim 10^{8} \mathrm{M}_{\odot}$. Moreover, our criterion identifies massive failures (relative to the M31 satellites) in Juliet (7) and in several of the isolated hosts: $\mathrm{m} 12 \mathrm{c}$ contains $6, \mathrm{~m} 12 \mathrm{i}$ contains 3 , and $\mathrm{m} 12 \mathrm{~m}$ contains 7 . We also remind the reader that the hydrodynamic versions of these haloes underproduce the SMFs; if this is due to the masses of our hosts, then we would expect to also underproduce the halo mass function, which scales closely with host mass (e.g. Boylan-Kolchin et al. 2010). Finally, both pairs contain a glut of unaccounted-for, massive haloes in their Local Field populations. Moreover, in both the pairs, at least two of those leftover haloes are too dense to be associated with any of the known galaxies other than Tucana or NGC 6822.

We emphasize that all of our DMO hosts (including those in the Latte suite) suffer from TBTF (as formulated by Garrison-Kimmel et al. 2014b) when comparing their satellite populations with the satellites of the MW. Though we only directly plot Juliet and Louise against the MW satellites, we list the number of massive failures (and, in parentheses, strong massive failures) in the final column of Table 1: in the DMO simulations, all of our hosts contain at least two strong massive failures.

\subsection{Results: FIRE simulations}

Fig. 5 is analogous to Fig. 4, but it plots $V_{\text {circ }}$ curves of the luminous galaxies in the FIRE simulations (i.e. including baryons). Because we colour the lines by stellar mass, we separate massive failures and haloes that are matched with observed dwarfs via line-style: massive failures are plotted with dashed lines and the haloes assigned to host galaxies with solid lines. The addition of baryonic physics to the simulations eliminates the TBTF problem around the MW and M31. In particular, there are neither 'strong massive failures' nor 'massive failures' within the virial radius of either host according to the definitions applied to the DMO simulations above. While the M31 population looks good in comparison to the TBTF problem, our hosts do not contain quite as many satellites as M31 overall: matching the SMF may result in additional galaxies that cannot be matched one-to-one with observed systems.

There do remain a number of 'failures', according to our formal definition in the Local Field population (dotted lines), all with stellar masses $<10^{6} \mathrm{M}_{\odot}$. However, we emphasize that their circular velocities are still much lower than in the DMO simulations; in fact, they have profiles quite similar to the typical observed systems in both the MW, M31, and Local Field. Given that the completeness of the Local Field out to $\sim \mathrm{Mpc}$ at these masses is rather uncertain, one possibility is that there is a population of $\sim 10$ undetected dwarf galaxies in this region, with stellar masses $M_{*}=10^{5-6} \mathrm{M}_{\odot}$ and dark matter densities similar to those of known dwarf galaxies (e.g. And XVIII). ${ }^{12}$ As noted above, the number of these galaxies may also be sensitive to the total mass of the Local Field, though our sample of two Local Fields displays a declining trend. We also note that this tension, like that in the Local Field SMF, can be reduced (decreasing the number of discrepant haloes by a few), without introducing significant tension in the comparison with TBTF around M31, if we use a larger radial cut as in Appendix B to associate galaxies with M31 and the MW. Furthermore, some of the measured circular velocities may not reflect the true dynamical mass. In particular, Lewis et al. (2007) found a significantly $(\sim 2 \times)$ higher dynamical mass for Cetus, and Oman et al. (2016) pointed out that IC 1613 may be rotating with an underestimated inclination angle, such that analyses underestimate the total mass.

Note that the relative impact of supernovae feedback is such that more massive dwarfs $\left(M_{*} \sim 10^{8} \mathrm{M}_{\odot}\right)$ almost universally have

\footnotetext{
${ }^{12}$ Specifically, there are 17 (7) of these missing systems in the Local Field of Romeo \& Juliet (Thelma \& Louise) with $M_{*}>10^{5} \mathrm{M}_{\odot}$ and 7 (6) with $M_{*}>3 \times 10^{5} \mathrm{M}_{\odot}$.
} 

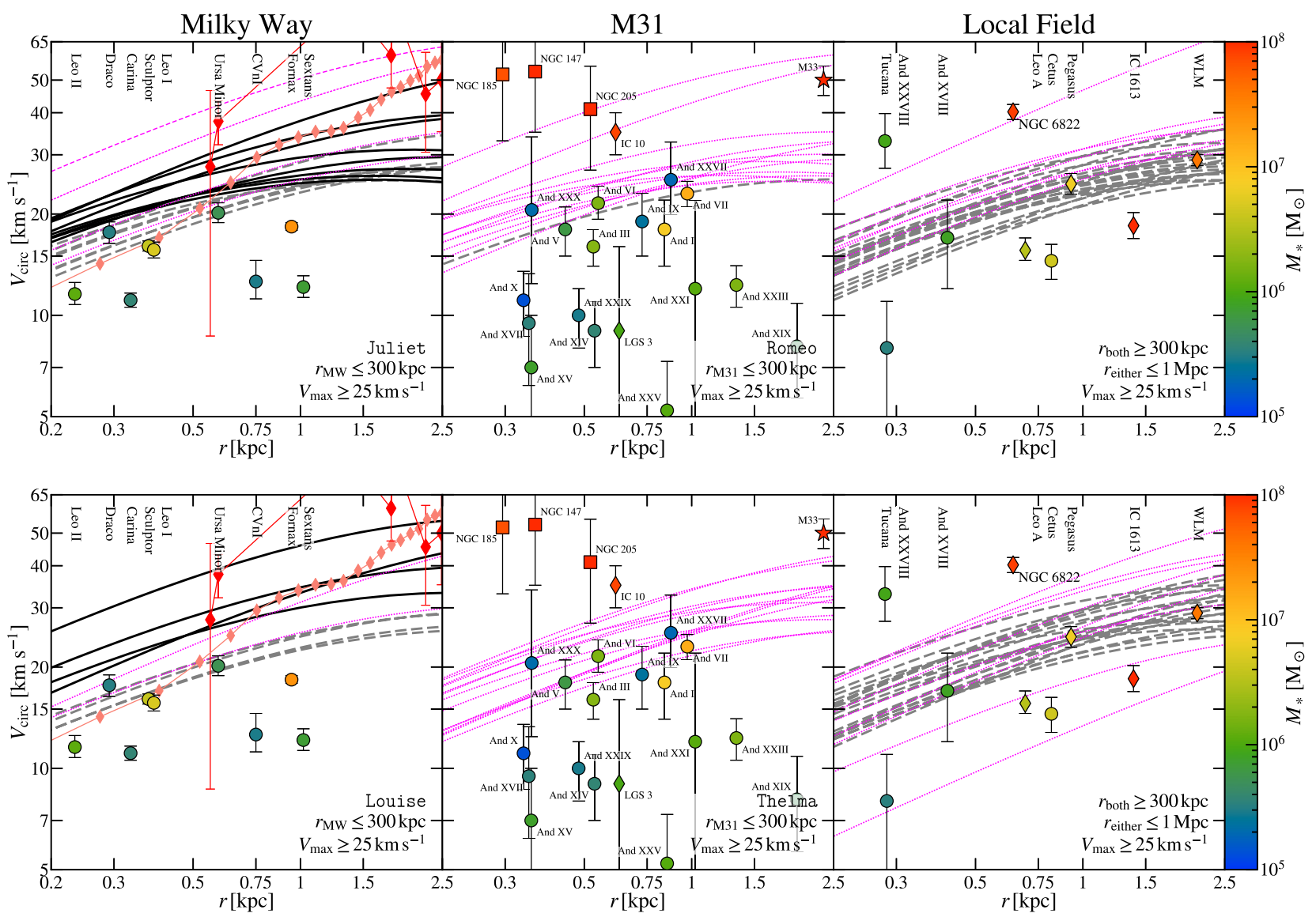

Figure 4. Circular velocity curves of dwarf (sub)haloes in the DMO simulations, selected according to $V_{\max }$, throughout Romeo \& Juli iet (top) and Thelma \& Louise (bottom). From left to right, the panels plot MW satellites, M31 satellites, and galaxies in the Local Field. The circles, squares, and diamonds represent dSphs, dEs, and dIrrs, respectively, with galaxy classifications taken from the literature; the star indicates M33, and the lines marked with diamonds indicate rotation curves for the SMC (small diamonds) and the LMC (large diamonds). 'Strong' massive failures, which are haloes too dense to host any of the galaxies in the comparison sample other than the LMC and SMC, are plotted as solid black lines. The less stringently defined massive failures, which are haloes expected to host relatively bright galaxies but that lack an observational counterpart, are plotted as dashed grey lines. Haloes assigned to host a galaxy are plotted in magenta. The subhaloes assigned to host the LMC and SMC (defined to be the two most massive, if they have $V_{\max } \geq 65$ and $60 \mathrm{~km} \mathrm{~s}^{-1}$, respectively) are plotted as short and long dashed magenta lines around Juliet. Both the M31 and the Local Field contain dwarfs that are dense enough to eliminate all strong massive failures and, when the dEs and M32 (outside the plot axes) are accounted for, typically only a few subhaloes with $V_{\max } \geq 25 \mathrm{~km} \mathrm{~s}^{-1}$ remain unaccounted for around M31. However, the TBTF problem, as identified by Boylan-Kolchin et al. $(2011,2012)$ around the MW and by Garrison-Kimmel et al. (2014b) in the Local Field, exists in the DMO simulations of all of our systems. Every host has several subhaloes that are too dense to host any of the MW dSphs, along with many more that are only consistent with Draco and Ursa Minor, and every Local Field analogue contains a plethora of massive subhaloes, many of which can only be associated with either Tucana or the baryon-dominated NGC 6822.

lower central masses than their less luminous counterparts $\left(M_{*} \lesssim\right.$ $\left.10^{6} \mathrm{M}_{\odot}\right)$, particularly in the Local Field. Measuring dynamical masses within $\sim 500$ pc across a range of stellar masses (e.g. with $30 \mathrm{~m}$-class telescopes) will test this prediction.

Aside from the Local Field, all of our hydrodynamic simulations (including the Latte suite) are free of TBTF: all of the simulated dwarf satellites are consistent with even the lower density MW $\mathrm{dSphs}$ and the satellites of M31. As we will show more quantitatively in Section 6, the stellar kinematics of the simulated galaxies are also in line with those of dwarfs throughout the LG.

The agreement between the central masses of the simulated and observed galaxies is not perfect, however: the satellite populations do not contain any systems quite as dense as NGC 205, NGC 147, NGC 185 , or IC $10 .{ }^{13}$ This result holds across our entire sample:

${ }^{13}$ They also do not contain any as dense as M32, but the high density of M32 may be at least partially explained by a nuclear supermassive black none of our hosts have satellites (or field galaxies) that reach even the lower $1 \sigma$ error on NGC 205, the least dense of the dEs. Though this may be due to a lack of high-mass dwarf galaxies, the trend is typically in the opposite direction, such that our high-mass dwarf galaxies have relatively low $V_{\text {circ }}$ at $\sim 300 \mathrm{pc}$. An examination of fig. 6 of Sawala et al. (2016b) and fig. 3 of Dutton et al. (2016) suggests that the APOSTLE and NIHAO simulations, respectively, may also lack analogues of the high-density M31 satellites (haloes with $V_{\text {circ }} \sim 50 \mathrm{~km} \mathrm{~s}^{-1}$ at $\sim 500 \mathrm{pc}$ ). These high-density galaxies may represent a manifestation of the 'diversity problem' (Oman et al. 2015; Creasey et al. 2017) in the LG.

Producing such high-density galaxies, with $M_{*} \sim 10^{8} \mathrm{M}_{\odot}$, may prove to be an important test of galaxy formation physics. In particular, while abundance matching arguments suggest that these 

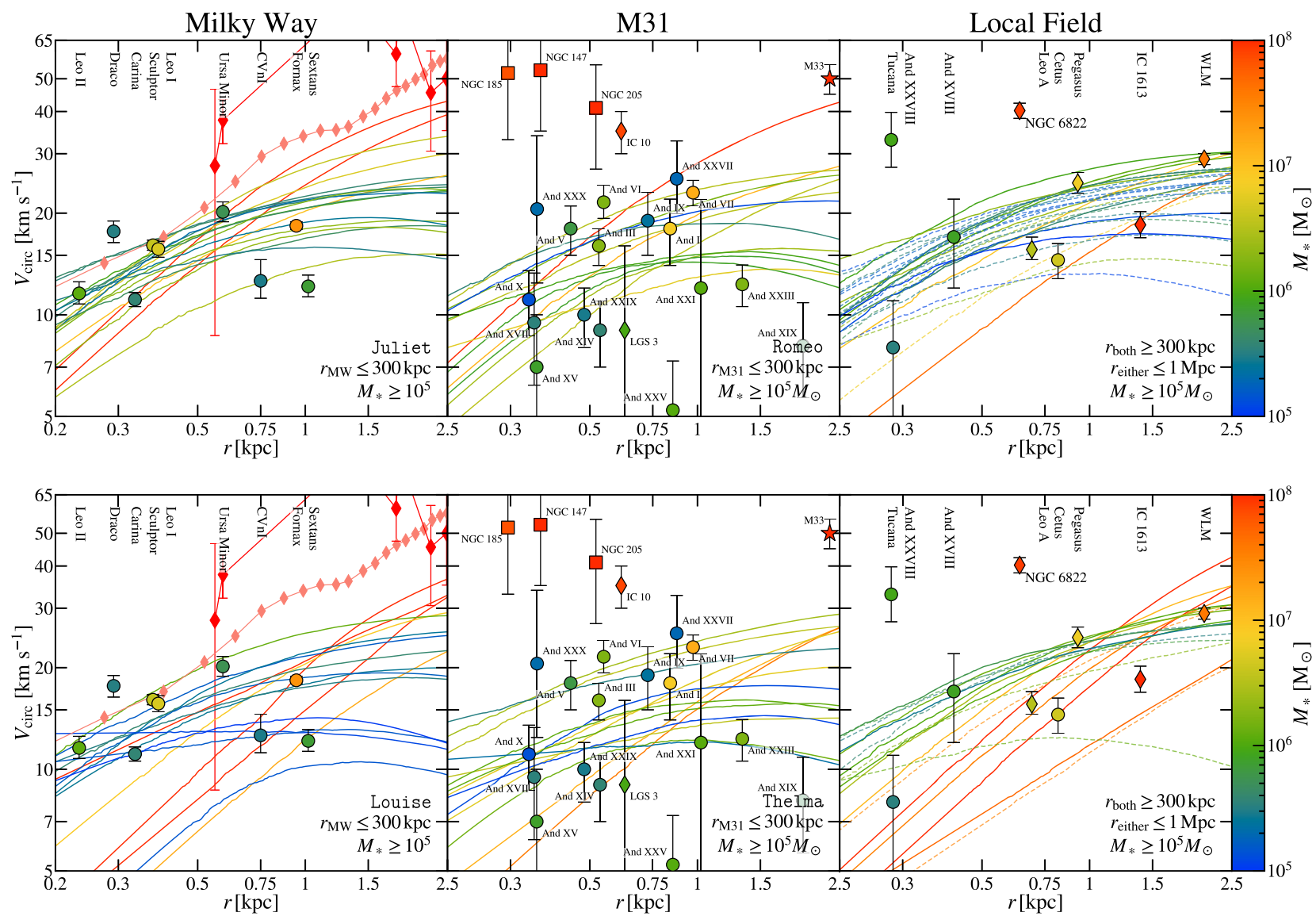

Figure 5. Identical to Fig. 4, but here plotting $V_{\text {circ }}$ curves from the hydrodynamic simulations. Including baryonic physics using the FIRE models eliminates TBTF around the MW and M31. The dotted lines in the Local Field panel show the persistence of several 'failures' unaccounted for by current data, but these are quite different from the massive failures in the DMO runs: they have rotation curves similar to the typical observed LG and Local Field systems (there are simply $\sim 10$ more of them). The mismatch may therefore be a result of observational incompleteness at $M_{*} \lesssim 10^{6} \mathrm{M}_{\odot}$. The simulations here do not produce any galaxies with densities as high as those of the baryon-dominated compact dEs around M31 (or Tucana/NGC 6822 ), with $V_{\text {circ }} \gtrsim 35 \mathrm{~km} \mathrm{~s}^{-1}$ at $r<1 \mathrm{kpc}$.

galaxies are at the centres of haloes that reached $\sim 10^{10.5} \mathrm{M}_{\odot}$ (Garrison-Kimmel et al. 2014a), previous work has shown that mass scale to be the most susceptible to core formation and stellar migration due to supernovae feedback (Di Cintio et al. 2014b; Chan et al. 2015; El-Badry et al. 2016). Some of these could be the stripped cores of previously more massive galaxies: for example, McConnachie et al. (2004) identified a stream that is likely originating from NGC 205. However, they estimate the total mass in that stream to be only $\sim 2.5$ per cent of the mass of NGC 205 . Moreover, this option is unlikely for at least IC 10, which is gas rich and star forming today. Furthermore, the galaxies in the LG that are more massive than this sample, the LMC and M33, lack these high-density central clumps. An additional, constant source of feedback (e.g. cosmic rays; Jubelgas et al. 2008) that acts to smooth out the burstiness in the star formation, leading to lessviolent feedback episodes, may be required to explain these objects. For a more detailed discussion of the structure of isolated galaxies at this mass scale in the FIRE-2 simulations, we refer the reader to Chan et al. (2017), who studied the evolution of the stellar effective radius; El-Badry et al. (2018b), who explored the gas morphologies as a function of galaxy mass; and El-Badry et al. (2018a), who showed that $M_{*} \sim 10^{8} \mathrm{M}_{\odot}$ galaxies are, on average, overly dispersion supported relative to spatially unresolved H I gas kinematics.
However, more detailed comparison of our existing simulations to these observations is also warranted, particularly to forwardmodel the actual observed rotation curves and velocity dispersions. Some of the observed systems with high apparent velocities are clearly tidally disturbed or strongly interacting (e.g. IC 10, Ashley et al. 2014, and NGC 205, above), and Teyssier, Johnston \& Kuhlen (2012) argue that NGC 147, 185, 6822, and Tucana all have had a previous passage through the MW or M31 disc. Some of these also feature recent starbursts, in which case El-Badry et al. (2017) argue that feedback-driven perturbations to the potential (the same that flattens the DM profile) can lead to the observationally inferred Jeans masses (hence $V_{\text {circ }}$ ) being overestimated by up to a factor of $\sim 2$ (sufficient to explain most of the discrepancy). We will show below, for example, that the actual line-of-sight stellar velocity dispersions in the simulations reach values similar to those observed even in the high-density systems.

\subsection{The impact of the shape of the density profile}

In summary, Figs 4 and 5 demonstrate that, while the DMO analogues to the ELVIS on FIRE simulations all suffer from TBTF, the problem is strongly alleviated or entirely eliminated in the fully hydrodynamic runs. Specifically, we find no TBTF problem around the MW analogues, a result consistent with observational 

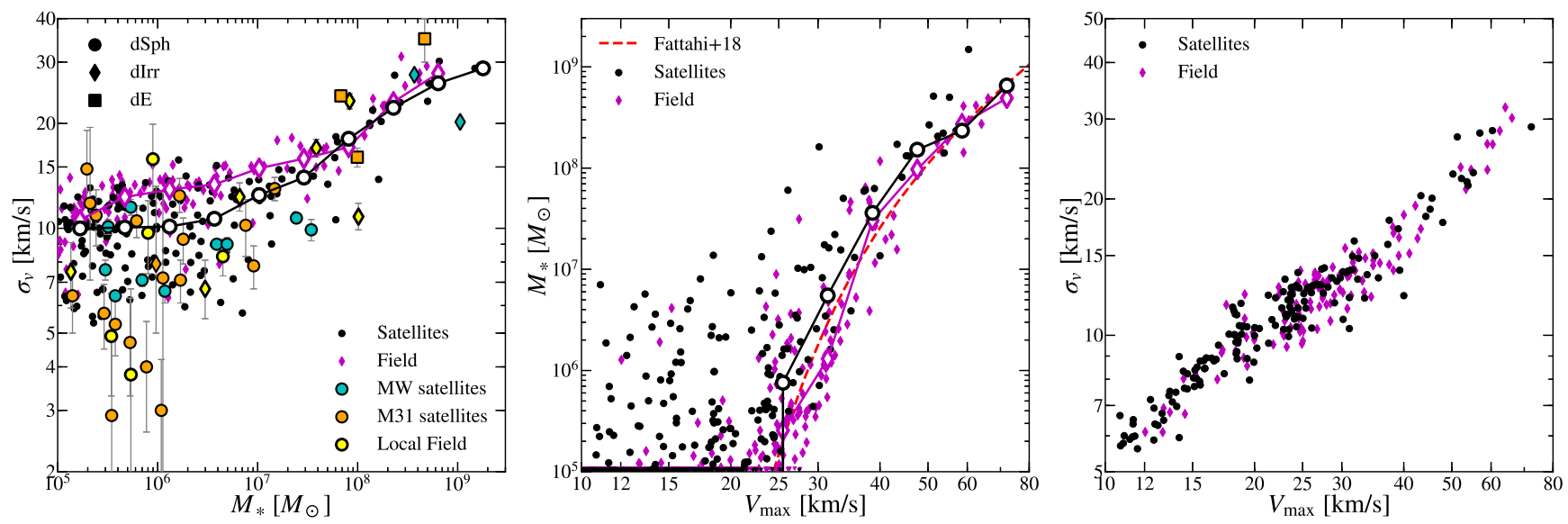

Figure 6. Relationships between stellar mass, 1D (line-of-sight) stellar velocity dispersion, and halo $V_{\max }$ including satellites and Local Field galaxies from all the simulations. The simulations generally reproduce the observed $M_{*}-\sigma_{*}$ relationship, particularly for the satellites of the MW, but they fail to create any dwarfs with $\sigma_{*}$ as low as some observed near M31, possibly because of either artificial destruction (specifically, an inability to track strongly tidally stripped objects) or $N$-body dynamical heating in the simulations. $V_{\max }$ is reasonably predictive of $M_{*}$ for non-satellite galaxies, but tidal interactions decrease $V_{\max }$ faster than $M_{*}$, generally scattering galaxies to the left. The red line in the centre panel plots the fit from Fattahi et al. (2018) to the population of dwarf galaxy centrals (non-satellites) in the APOSTLE simulations, which agrees well with the median relation for our non-satellite objects. $V_{\max }$ and $\sigma_{*}$ remain remarkably correlated, however. The open points in the left two panels indicate the medians for each population. The downward arrows in the central panel indicate haloes that fall off the plot (i.e. $M_{*}<10^{5} \mathrm{M}_{\odot}$ ), which first appear for $V_{\max } \lesssim 25 \mathrm{~km} \mathrm{~s}^{-1}$ and become common at $V_{\max } \lesssim 20 \mathrm{~km} \mathrm{~s}^{-1}$. We do not claim that these haloes are necessarily 'dark', merely that they are at lower stellar mass. For the purposes of calculating the medians in each population, these points are treated as having a stellar mass of zero.

incompleteness in the Local Field, and a set of dwarf galaxies consistent with the dSphs around M31 (though we find no analogues to the higher density satellites of M31).

However, the analysis above was performed with two caveats: first, we assume NFW profiles for the DMO (sub)haloes but calculate $V_{\text {circ }}$ for the FIRE simulations by joining fitted density profiles to the raw particle data, and secondly, we compare only the lower mass host in each pair to the MW dSphs. The second choice has no effect on our results: by the metrics defined above, none of our hosts, paired or isolated, have any massive failures in their luminous satellites when compared with the MW dSphs.

The first choice is similarly irrelevant to our conclusions, but it does have relatively large consequences for the number of 'strong' massive failures identified in the satellite populations of the DMO simulations: without correcting for the numerical impact of gravitational softening, we identify only 11 strong massive failures across the 10 DMO hosts, compared with 61 when we assume NFW profiles. The number of massive failures in the DMO runs, however, is much more stable to this assumption and only decreases by 1-4 in all but two of our hosts, with the total count decreasing by only 31 per cent from 114 to 79 . That is, by the Garrison-Kimmel et al. (2014b) metrics, we would still have identified a TBTF problem, even drawing directly from the particle data. We also emphasize that the assumption of NFW (or NFW-like) profiles for the DMO subhaloes is theoretically well motivated. Nevertheless, we plot the raw DMO $V_{\text {circ }}$ curves for Romeo \& Juliet in Appendix B for illustrative purposes.

More importantly, the results for the FIRE simulations are also only weakly sensitive to the shape of the central density profile. Specifically, adopting a cuspy NFW profile versus using the corrected (or raw) mass profile has a relatively minor influence on the number of massive failures identified in the FIRE simulations, particularly when compared with the MW satellites. Assuming NFW profiles for the luminous satellites in the hydrodynamic runs (similarly normalized to $R_{\max }$ and $V_{\max }$ of each subhalo) yields a total of only 13 massive failures across our 10 hosts when compared with the MW dSph sample, only three of which are 'strong'.

Therefore, even though there is now substantial evidence that supernovae feedback can flatten the central density profiles of $M_{*} \simeq 10^{6.5}-10^{9} \mathrm{M}_{\odot}$ galaxies (e.g. Pontzen \& Governato 2012; Di Cintio et al. 2014a; Chan et al. 2015) we find that this effect is typically of second-order importance for solving TBTF among the satellite populations in these simulations (in agreement with Sawala et al. 2016b). Instead, the problem is primarily alleviated by removing mass from the subhaloes overall (lowering $V_{\max }$ ) and destroying otherwise luminous satellites through enhanced tidal interactions with the disc (D'Onghia et al. 2010; Garrison-Kimmel et al. 2017; Sawala et al. 2017). However, we cannot completely dismiss the importance of feedback-induced core formation; for example, subhaloes cored by internal processes are then more susceptible to further mass-loss from external interactions (e.g. Peñarrubia et al. 2010; Brooks \& Zolotov 2014, but also see Garrison-Kimmel et al. 2017, who showed that much of the differences in subhalo counts between DMO and FIRE simulations can be accounted for purely by the gravitational potential of the central galaxy with only a weak dependence on subhalo mass or $\left.V_{\max }\right)$.

Changes to the internal profile are also relatively unimportant in the Local Field, even though tidal effects are minimal in that volume: assuming NFW profiles for the non-satellite sample within $1 \mathrm{Mpc}$ of each host increases the total number of massive failures (defined in this volume as galaxies with $M_{*} \geq 10^{5} \mathrm{M}_{\odot}$ without observational kinematic counterparts) across the entire simulated sample from 34 to 40 . However, this difference is still small compared to the overall impact of baryonic physics: the same volumes contain $\simeq 75$ haloes identified as massive failures when simulated without baryons (nearly independent of whether we assume NFW profiles or use the raw particle data). Therefore, even in the Local Field, 
feedback-induced cores are only a small piece of resolving TBTF: overall baryonic mass-loss, enhanced disruption (both from other field galaxies and in the sample of 'backsplash' haloes), and changes to the halo sample due to selecting on $M_{*}$ rather than $V_{\max }$ all play a significant role, even for non-satellite galaxies.

\section{STELLAR VELOCITY DISPERSIONS}

We have shown separately that the distributions of stellar masses and rotation curves of our simulated dwarf populations broadly agree with that of the LG. One can additionally ask whether our simulations predict the correct joint relation between these, that is, whether our individual dwarf galaxies are indeed realistic. Fig. 6 directly compares the stellar velocity dispersions (defined as the RMS line-of-sight dispersion of all the stars associated with a galaxy) as a function of stellar mass for all of the satellite galaxies (defined as $r<300 \mathrm{kpc}$ ) and non-satellite galaxies in the simulations, together with dwarf galaxies from throughout the LG. Though $\sigma_{*}$ is not necessarily representative of the underlying DM halo (e.g. in the case of significant rotation, such as for the LMC, the rightmost point in the plot), the overall agreement between the simulated and observed relationships supports our assertion that our dwarf galaxy populations display similar kinematics as the observed LG dwarf galaxies.

However, the simulations fail to reproduce the six LG galaxies with $M_{*}>3 \times 10^{5} \mathrm{M}_{\odot}$ and $\sigma_{*} \leq 5 \mathrm{~km} \mathrm{~s}^{-1}$, all of which are within $400 \mathrm{kpc}$ of M31. This disagreement may indicate that our resolution (for the stars, gravitational softening lengths $\lesssim 5 \mathrm{pc}$ and particle masses $\lesssim 10^{3.5}$ ) remains insufficient for resolving the coldest, and potentially most disrupted, dwarf galaxies in the LG; these systems have $\lesssim 100$ star particles in the simulations. The worst-case velocity kick (i.e. the maximal possible deflection) due to $\mathrm{N}$-body interactions between stellar particles is of the order of $3 \mathrm{~km} \mathrm{~s}^{-1}$ in our simulations. Therefore, it may not be possible to maintain systems as dynamically cold as these six galaxies. There is also evidence for a partial separation between the satellite and non-satellite populations, such that satellite galaxies lose dynamical mass and scatter to lower $\sigma_{*}$ at fixed $M_{*}$. If, as suggested by Brooks \& Zolotov (2014), Zolotov et al. (2012), and Fattahi et al. (2018), this is due to tidal effects, then the simulated analogues of the outlying galaxies in Fig. 6 may be (spuriously) destroyed due to finite mass resolution (van den Bosch \& Ogiya 2018). However, those authors demonstrated that artificial numerical disruption could be minimized with aggressive gravitational force softenings, and we remind the reader that our simulations adopt physical DM force softenings of $\simeq 50 \mathrm{pc}$.

We also note that, while we do not plot it, our simulations typically agree reasonably well with the $R_{1 / 2}$ distribution of the LG population at fixed $M_{*}$ or $\sigma_{*}$, but they do not reproduce the spatially smallest/most compact systems at a given $M_{*}$ and are larger, on average, at either fixed $M_{*}$ or fixed $\sigma_{*}$. The results of even higher resolution FIRE simulations of isolated dwarf galaxies suggest that our smallest simulated dwarfs $\left(M_{*} \lesssim 10^{6} \mathrm{M}_{\odot}\right)$ will likely become more compact with increased resolution (Fitts et al. 2017), but higher mass dwarf galaxies simulated with FIRE maintain large effective radii even for gas particle masses $260 \mathrm{M}_{\odot}$ (Chan et al. 2017) as their sizes are set by feedback 'puffing up' the system. Given the insensitivity of our results to the internal profiles of the simulated satellites, we do not expect that increasing the resolution will significantly alter our conclusions with respect to TBTF. Moreover, in lower resolution FIRE simulations, the higher mass (i.e. resolved) dwarf galaxies also yield a reasonable $M_{*}-$ $\sigma_{*}$ relationship. However, our overly extended galaxies may also contain more dark matter (if the dark matter density profile is independent of $R_{1 / 2}$ ), which would tend to increase $\sigma_{*}$ at a given $M_{*}$. Our dwarfs may also be more susceptible to tidal stripping due to their large sizes, though the results of Garrison-Kimmel et al. (2017) and Kelley et al. (2018) suggest that the survival of a subhalo is primarily dependent on its orbit, given that the survival rate of subhaloes with pericentric distances $\lesssim 25 \mathrm{kpc}$ is effectively zero when including the gravitational potential of the MW disc.

The right two panels in Fig. 6 plot the stellar mass and stellar velocity dispersion as a function of $V_{\max }$. The relationship between $M_{*}$ and $V_{\max }$ is relatively tight for isolated galaxies, where $V_{\max }$ is more likely to represent the largest mass the halo ever reached, but it is clear that tidal interactions shift galaxies to the left on the plot by removing dark matter from the outer portions of the subhaloes, decreasing $V_{\max }$ faster than $M_{*} \cdot{ }^{14}$ Both results are in good agreement with the results of the APOSTLE simulations (Sawala et al. 2016b; Fattahi et al. 2018). The dashed red line in the centre panel shows the fit from the latter publication to the centrals in those simulations; it agrees remarkably well with the median of our nonsatellite sample. Meanwhile, the relationship between $V_{\max }$ and $\sigma_{*}$ remains remarkably tight even after tidal interactions with a larger halo.

The downward arrows at the bottom of the centre panel indicate haloes with $M_{*}<10^{5} \mathrm{M}_{\odot}$, i.e. that fall below the $y$-limit of the plot. Our analysis assigns the vast majority of these haloes no stars, though a few contain a small number of star particles. These systems begin to appear for $V_{\max } \lesssim 25 \mathrm{~km} \mathrm{~s}^{-1}$ and become frequent for $V_{\max }$ $\lesssim 20 \mathrm{~km} \mathrm{~s}^{-1}$. These results are in rough agreement with Sawala et al. (2016a) and Benítez-Llambay et al. (2017): the fraction of haloes in these simulations that fall below $M_{*}=10^{5} \mathrm{M}_{\odot}-$ but which are not necessarily dark - reaches 50 per cent at $V_{\max } \simeq$ $24 \mathrm{~km} \mathrm{~s}^{-1}$ for non-satellites and $\simeq 20 \mathrm{~km} \mathrm{~s}^{-1}$ for satellites. If these haloes host ultra-faint dwarf galaxies below our resolution limits, then such galaxies should appear to be fairly dense, with central masses similar to And XVIII. Because our definition of 'massive failure' includes only haloes with $V_{\max } \geq 25 \mathrm{~km} \mathrm{~s}^{-1}$, these dark haloes contribute only marginally towards resolving TBTF because the fraction of haloes with $M_{*} \leq 10^{5} \mathrm{M}_{\odot}$ is only $\sim 15$ per cent at $V_{\max }=25 \mathrm{~km} \mathrm{~s}^{-1}$, and only $\sim 5$ per cent of haloes with $V_{\max } \geq$ $25 \mathrm{~km} \mathrm{~s}^{-1}$ having $M_{*} \leq 10^{5} \mathrm{M}_{\odot}$. However, the values plotted in Fig. 6 are taken from the hydrodynamic simulations; it is therefore possible that DMO haloes with $V_{\max } \gtrsim 25 \mathrm{~km} \mathrm{~s}^{-1}$ accreted less overall mass in the hydrodynamic simulations and appear as dark haloes with $V_{\max } \lesssim 20 \mathrm{~km} \mathrm{~s}^{-1}$.

\section{CONCLUSIONS}

The LG provides an unparalleled window into the population of dwarf galaxies in the Universe, but it is not a typical environment: the presence of two massive haloes $\left(\gtrsim 10^{12} \mathrm{M}_{\odot}\right)$ has important implications for, e.g. the predicted halo mass function in the nearby volume (Garrison-Kimmel et al. 2014a). Here, we present

\footnotetext{
${ }^{14}$ The relationship between $M_{*}$ and $V_{\max }$ for non-satellite galaxies is in stark contrast to the findings of Garrison-Kimmel et al. (2014b), who found no trend between $M_{*}$ and the implied $V_{\max }$ for galaxies in the Local Field. However, that analysis assumed fixed density profiles across all haloes, and assigned $V_{\max }$ by extrapolating from $V_{1 / 2}$. An updated analysis that accounts for variance in the density profiles as a function of $M_{*}$ and $M_{\mathrm{vir}}$ is required to properly assign $V_{\max }$ values to the Local Field systems.
} 
the first two simulations from the ELVIS on FIRE suite, which apply the FIRE models for star formation and feedback to LGlike environments at $\lesssim 4000 \mathrm{M}_{\odot}$ resolution. We also include results from FIRE simulations targeting isolated MW-mass haloes at similar resolutions. We present the satellite and non-satellite SMFs predicted by these simulations, and compare them to an analogous set of isolated MW-mass haloes also simulated with FIRE. We then compare the internal structure of our resolved galaxies to that of the dwarf galaxies in the LG, both via their implied dynamical masses within the half-light radius (the TBTF problem) and through the relationships between $\sigma_{*}$ and $M_{*}$.

The simulations accurately reproduce the dwarf galaxy population of the MW for $M_{*} \gtrsim 10^{5} \mathrm{M}_{\odot}$. They roughly bracket the SMF of the MW satellites at nearly all masses, particularly below the masses of the LMC and SMC. However, the MW SMF is unique in exhibiting a 'gap' between $\mathrm{CVnI}\left(M_{*}=3 \times 10^{5} \mathrm{M}_{\odot}\right)$ and the ultra-faint dwarfs with $M_{*} \lesssim 3 \times 10^{4} \mathrm{M}_{\odot}$, suggesting observational incompleteness around the MW even for $M_{*}>10^{5} \mathrm{M}_{\odot}$ (typically $\approx 4$ such galaxies). The simulated satellite galaxies also have central masses consistent with those of the real MW satellites: they do not suffer from TBTF. This result is relatively insensitive to the shape of the central density profile, particularly compared to the total impact of baryonic physics: even if we (falsely) assume a cuspy, NFW profile for the hydrodynamic simulations, we identify less than two 'massive failures' per host on average, while the DMO simulations contain more than 11 . Therefore, supernova-induced core formation is less important in resolving TBTF among the MW satellites: subhalo disruption and overall mass-loss appear to be the dominant processes.

Our simulated satellites are somewhat less successful at reproducing the population of dwarf galaxies around M31. They (usually) underproduce the total count at most stellar masses: M31 contains, on average, roughly twice as many satellites with $M_{*} \geq 10^{5} \mathrm{M}_{\odot}$ as the median simulated host. Given that the highest mass host in our sample has $M_{\mathrm{vir}}=1.54 \times 10^{12} \mathrm{M}_{\odot}$, this may suggest a higher virial mass for M31. However, we find only a weak trend between host halo mass and the number of satellites above an $M_{*}$. Moreover, while our simulated satellites have central masses consistent with the dSphs around M31, none of our dwarf galaxies appears to have enough mass within $\sim 300$ pc to host the highest density dwarf galaxies inferred around M31 (the three dEs and IC 10) - the opposite problem as TBTF. Our simulations may also lack the resolution to reproduce the six dwarf galaxies within $400 \mathrm{kpc}$ of M31 with $\sigma_{*}<5 \mathrm{~km} \mathrm{~s}^{-1}$ and $M_{*} \geq 3 \times 10^{5} \mathrm{M}_{\odot}$. More detailed modelling to predict the kinematics that would actually be measured in both these cases is clearly warranted.

The simulated non-satellite $\left(r_{\text {host }}>300 \mathrm{kpc}\right)$ populations agree reasonably well with the observations: they again roughly bracket the observed SMFs, now for $M_{*} \geq 10^{6} \mathrm{M}_{\odot}$, and have central masses that are consistent with observations of the majority of the dwarf galaxies in the Local Field. However, while the TBTF problem is resolved for satellite systems around the MW and M31, the simulations predict the existence of $\sim 10$ low-mass dwarf galaxies within $\sim 1 \mathrm{Mpc}$ of each host that are currently unaccounted for in the data, though there is significant system-to-system scatter. These all have $M_{*}=10^{5}-10^{6} \mathrm{M}_{\odot}$, and circular velocities broadly similar to those observed in other LG and Local Field dwarfs of the same mass, and thus may represent an as-of-yet undetected population of low-mass dwarf galaxies in the Local Field. This prediction should be testable with a combination of LSST, WFIRST, and $30 \mathrm{~m}-$ class telescopes. However, we note that both this discrepancy and that with the M31 SMF may be quantitatively reduced if some of our 'Local Field' population should really be associated with M31 (in observations), and our non-paired haloes demonstrate large systematic scatter in their field SMFs.

Other than the very low $\sigma_{*}$ dwarf galaxies near M31, our simulated dwarfs broadly overlap the observations in $M_{*}$ versus $\sigma_{*}$. We find a tight relationship between $\sigma_{*}$ and $V_{\max }$ for both satellites and non-satellites. The relationship between $V_{\max }$ and $M_{*}$ is also relatively tight for non-satellites, but tidal interactions introduce substantial scatter among the satellite populations.

In short, neither the isolated, MW-mass FIRE simulations nor the ELVIS on FIRE simulations suffer from the traditional small-scale problems identified for satellites within the virial radius of the MW or M31. Further, the ELVIS on FIRE simulations alleviate the TBTF problem in the Local Field, though there remains some tension that needs to be tested with future observations.

Our simulations are not free of flaws. They ignore some physical processes that may be important at these scales (e.g. supermassive black holes and cosmic rays), they include a reionization history that is on the early edge of constraints from Planck (Oñorbe, Hennawi \& Lukić 2017), they appear to lack the necessary resolution to capture the half-mass radii of the smallest galaxies $\left(M_{*} \lesssim 10^{6} \mathrm{M}_{\odot}\right)$, and they may fail to reproduce the highest density dwarf galaxies in the LG. Given that supernovae feedback appears to be most effective at these mass scales, their existence may point towards physics that reduces the burstiness in star formation (lessening the violent feedback episodes associated with strong bursts). Altogether, however, our results indicate that a meta-galactic ionizing background, stellar/supernovae feedback, and interactions with the discs of the MW and M31 are able to transform the overly abundant, overly dense LG (sub)halo populations predicted by DMO simulations into a sample of dwarf galaxies that is largely consistent with observations of the LG within the vanilla $\Lambda$ CDM paradigm, though our work does not rule out non-standard DM physics. Future work is required to fully understand the relative contributions of internal feedback, LG-scale interactions, and the cosmological background to dwarf galaxy formation in the LG, to test the impact of host mass on the satellite populations, and to understand the formation of the high-density dEs in the LG.

\section{ACKNOWLEDGEMENTS}

The authors thank Evan Kirby, Coral Wheeler, Lina Necib, Alejandro Benitez-Llambay, and Cameron Hummels for valuable discussions, and Alexander Knebe and Oliver Hahn for making AHF and MUSIC, respectively, publicly available.

Support for SGK was provided by NASA through Einstein Postdoctoral Fellowship grant number PF5-160136 awarded by the Chandra X-ray Center, which is operated by the Smithsonian Astrophysical Observatory for NASA under contract NAS8-03060. Support for PFH was provided by an Alfred P. Sloan Research Fellowship, NSF Collaborative Research Grant \#1715847 and CAREER grant \#1455342. AW was supported by a CaltechCarnegie Fellowship, in part through the Moore Center for Theoretical Cosmology and Physics at Caltech, and by NASA through grants HST-GO-14734 and HST-AR-15057 from STScI. JSB was supported by NSF grant AST-1518291 and by NASA through HST theory grants (programs AR-13921, AR-13888, and AR14282.001) awarded by STScI, which is operated by the Association of Universities for Research in Astronomy (AURA), Inc., under NASA contract NAS5-26555. MBK acknowledges support from NSF grant AST-1517226 and CAREER grant AST-1752913 and from NASA grants NNX17AG29G and HST-AR-13888, HST-AR- 
13896, HST-AR-14282, HST-AR-14554, HST-AR-15006, HSTGO-12914, and HST-GO-14191 from STScI. DK was supported by NSF grant AST-1715101 and the Cottrell Scholar Award from the Research Corporation for Science Advancement. CAFG was supported by NSF through grants AST-1412836, AST-1517491, AST-1715216, and CAREER award AST-1652522, by NASA through grant NNX15AB22G, and by a Cottrell Scholar Award from the Research Corporation for Science Advancement. KEB was supported by a Berkeley graduate fellowship, a Hellman award for graduate study, and a NSF Graduate Research Fellowship. EQ was supported in part by NSF grant AST-1715070 and a Simons Investigator Award from the Simons Foundation. RS is supported by a NSF Astronomy \& Astrophysics Postdoctoral Fellowship under grant AST-1400989.

Numerical calculations were run on the Caltech compute cluster 'Wheeler', allocations from XSEDE TG-AST130039 and PRAC NSF.1713353 supported by the NSF, NASA HEC SMD-16-7223 and SMD-16-7592, and High Performance Computing at Los Alamos National Labs. This work also made use of Astropy, a community-developed core Python package for Astronomy (Astropy Collaboration 2013), matplotlib (Hunter 2007), numpy (van der Walt, Colbert \& Varoquaux 2011), scipy (Jones et al. 2001), ipython (Perez \& Granger 2007), yt (Turk et al. 2011), and NASA's Astrophysics Data System. This research was supported in part by the National Science Foundation under Grant No. NSF PHY-1748958.

\section{REFERENCES}

Ackermann M. et al., 2015, Phys. Rev. Lett., 115, 231301

Ashley T., Elmegreen B. G., Johnson M., Nidever D. L., Simpson C. E., Pokhrel N. R., 2014, AJ, 148, 130

Astropy Collaboration, 2013, A\&A, 558, A33

Behroozi P. S., Wechsler R. H., Wu H.-Y., 2013, ApJ, 762, 109

Bellazzini M., Gennari N., Ferraro F. R., 2005, MNRAS, 360, 185

Bellazzini M., Gennari N., Ferraro F. R., Sollima A., 2004, MNRAS, 354, 708

Bell E. F., de Jong R. S., 2001, ApJ, 550, 212

Bell E. F., Slater C. T., Martin N. F., 2011, ApJ, 742, L15

Belokurov V. et al., 2009, MNRAS, 397, 1748

Benítez-Llambay A. et al., 2017, MNRAS, 465, 3913

Bernard E. J. et al., 2009, ApJ, 699, 1742

Bernard E. J. et al., 2010, ApJ, 712, 1259

Bland-Hawthorn J., Gerhard O., 2016, ARA\&A, 54, 529

Bonanos A. Z., Stanek K. Z., Szentgyorgyi A. H., Sasselov D. D., Bakos G. Á., 2004, AJ, 127, 861

Bose S., Deason A. J., Frenk C. S., 2018, ApJ, 863, 123

Boylan-Kolchin M., Bullock J. S., Kaplinghat M., 2011, MNRAS, 415, L40

Boylan-Kolchin M., Bullock J. S., Kaplinghat M., 2012, MNRAS, 422, 1203

Boylan-Kolchin M., Springel V., White S. D. M., Jenkins A., 2010, MNRAS, 406, 896

Brasseur C. M., Martin N. F., Rix H.-W., Irwin M., Ferguson A. M. N., McConnachie A. W., de Jong J., 2011, ApJ, 729, 23

Brooks A. M., Zolotov A., 2014, ApJ, 786, 87

Bryan G. L., Norman M. L., 1998, ApJ, 495, 80

Buck T., Macciò A. V., Dutton A. A., Obreja A., Frings J., 2019, MNRAS, 483,1314

Bullock J. S., Boylan-Kolchin M., 2017, ARA\&A, 55, 343

Bullock J. S., Kravtsov A. V., Weinberg D. H., 2000, ApJ, 539, 517

Busha M. T., Wechsler R. H., Behroozi P. S., Gerke B. F., Klypin A. A., Primack J. R., 2011, ApJ, 743, 117

Carlesi E., Hoffman Y., Sorce J. G., Gottlöber S., Yepes G., Courtois H., Tully R. B., 2016, MNRAS, 460, L5

Carrera R., Aparicio A., Martínez-Delgado D., Alonso-García J., 2002, AJ, 123,3199
Chan T. K., Kereš D., Oñorbe J., Hopkins P. F., Muratov A. L., FaucherGiguère C.-A., Quataert E., 2015, MNRAS, 454, 2981

Chan T. K., Kereš D., Wetzel A., Hopkins P. F., Faucher-Giguère C.-A., El-Badry K., Garrison-Kimmel S., Boylan-Kolchin M., 2017, 478, 906 Chapman S. C. et al., 2013, MNRAS, 430, 37

Clementini G., Gratton R., Bragaglia A., Carretta E., Di Fabrizio L., Maio M., 2003, AJ, 125, 1309

Collins M. L. M. et al., 2010, MNRAS, 407, 2411

Collins M. L. M. et al., 2013, ApJ, 768, 172

Conn A. R. et al., 2012, ApJ, 758, 11

Cook K. H., Mateo M., Olszewski E. W., Vogt S. S., Stubbs C., Diercks A., 1999, PASP, 111, 306

Corbelli E., 2003, MNRAS, 342, 199

Corbelli E., Salucci P., 2000, MNRAS, 311, 441

Crain R. A. et al., 2015, MNRAS, 450, 1937

Creasey P., Sameie O., Sales L. V., Yu H.-B., Vogelsberger M., Zavala J., 2017, MNRAS, 468, 2283

Dale D. A. et al., 2007, ApJ, 655, 863

de Jong J. T. A. et al., 2008, ApJ, 680, 1112

de Vaucouleurs G., de Vaucouleurs A., Corwin H. G., Jr, Buta R. J., Paturel G., Fouqué P., 1991, Third Reference Catalogue of Bright Galaxies. Volume I: Explanations and references. Volume II: Data for galaxies between $0^{h}$ and $12^{h}$. Volume III: Data for galaxies between $12^{h}$ and $24^{h}$, Vol. 82, NO. 6/DEC. Sky \& Telescope, p. 621

Diaz J. D., Koposov S. E., Irwin M., Belokurov V., Evans N. W., 2014 MNRAS, 443, 1688

Di Cintio A., Brook C. B., Dutton A. A., Macciò A. V., Obreja A., Dekel A., 2017, MNRAS, 466, L1

Di Cintio A., Brook C. B., Dutton A. A., Macciò A. V., Stinson G. S., Knebe A., 2014b, MNRAS, 441, 2986

Di Cintio A., Brook C. B., Macciò A. V., Stinson G. S., Knebe A., Dutton A. A., Wadsley J., 2014a, MNRAS, 437, 415

Drlica-Wagner A. et al., 2015, ApJ, 813, 109

Dutton A. A., Macciò A. V., Frings J., Wang L., Stinson G. S., Penzo C., Kang X., 2016, MNRAS, 457, L74

D’Onghia E., Springel V., Hernquist L., Keres D., 2010, ApJ, 709, 1138

El-Badry K., Wetzel A., Geha M., Hopkins P. F., Kereš D., Chan T. K., Faucher-Giguère C.-A., 2016, ApJ, 820, 131

El-Badry K., Wetzel A. R., Geha M., Quataert E., Hopkins P. F., Kereš D., Chan T. K., Faucher-Giguère C.-A., 2017, ApJ, 835, 193

El-Badry K. et al., 2018a, MNRAS, 477, 1536

El-Badry K. et al., 2018b, MNRAS, 473, 1930

Escala I. et al., 2018, MNRAS, 474, 2194

Fattahi A., Navarro J. F., Frenk C. S., Oman K. A., Sawala T., Schaller M., 2018, MNRAS, 476, 3816

Fattahi A., Navarro J. F., Sawala T., Frenk C. S., Sales L. V., Oman K., Schaller M., Wang J., 2016a, preprint (arXiv e-prints)

Fattahi A. et al., 2016b, MNRAS, 457, 844

Faucher-Giguère C.-A., 2018, MNRAS, 473, 3717

Fiorentino G. et al., 2010, ApJ, 708, 817

Fitts A. et al., 2017, MNRAS, 471, 3547

Fraternali F., Tolstoy E., Irwin M. J., Cole A. A., 2009, A\&A, 499, 121

Frinchaboy P. M., Majewski S. R., Muñoz R. R., Law D. R., Łokas E. L., Kunkel W. E., Patterson R. J., Johnston K. V., 2012, ApJ, 756, 74

Garrison-Kimmel S., Boylan-Kolchin M., Bullock J. S., Kirby E. N., 2014b, MNRAS, 444, 222

Garrison-Kimmel S., Boylan-Kolchin M., Bullock J. S., Lee K., 2014a, MNRAS, 438, 2578

Garrison-Kimmel S., Horiuchi S., Abazajian K. N., Bullock J. S., Kaplinghat M., 2014c, MNRAS, 444, 961

Garrison-Kimmel S. et al., 2017, MNRAS, 471, 1709

Garrison-Kimmel S. et al., 2018, MNRAS, 481, 4133

Geha M., Guhathakurta P., Rich R. M., Cooper M. C., 2006, AJ, 131, 332

Geha M., van der Marel R. P., Guhathakurta P., Gilbert K. M., Kalirai J., Kirby E. N., 2010, ApJ, 711, 361

Gieren W., Pietrzyński G., Nalewajko K., Soszyński I., Bresolin F., Kudritzki R.-P., Minniti D., Romanowsky A., 2006, ApJ, 647, 1056

Gottloeber S., Hoffman Y., Yepes G., 2010, preprint (arXiv:1005.2687) 
Grand R. J. J. et al., 2017, MNRAS, 467, 179

Hahn O., Abel T., 2011, MNRAS, 415, 2101

Harris J., Zaritsky D., 2006, AJ, 131, 2514

Hernquist L., 1990, ApJ, 356, 359

Ho N. et al., 2012, ApJ, 758, 124

Hopkins P. F., 2015, MNRAS, 450, 53

Hopkins P. F., 2017, MNRAS, 466, 3387

Hopkins P. F., Kereš D., Oñorbe J., Faucher-Giguère C.-A., Quataert E., Murray N., Bullock J. S., 2014, MNRAS, 445, 581

Hopkins P. F., Narayanan D., Murray N., 2013, MNRAS, 432, 2647

Hopkins P. F. et al., 2018, MNRAS, 480, 800

Howley K. et al., 2012, preprint (arXiv:1202.2897)

Hunter D. A., Elmegreen B. G., 2006, ApJS, 162, 49

Hunter J. D., 2007, Comput. Sci. Eng., 9, 90

Ibata R., Martin N. F., Irwin M., Chapman S., Ferguson A. M. N., Lewis G. F., McConnachie A. W., 2007, ApJ, 671, 1591

Jaffe W., 1983, MNRAS, 202, 995

Jones E. et al., 2001, SciPy: Open source scientific tools for Python. http: //www.scipy.org/

Jubelgas M., Springel V., Enßlin T., Pfrommer C., 2008, A\&A, 481, 33

Kafle P. R., Sharma S., Lewis G. F., Robotham A. S. G., Driver S. P., 2018, MNRAS, 475, 4043

Katz N., White S. D. M., 1993, ApJ, 412, 455

Kelley T., Bullock J. S., Garrison-Kimmel S., Boylan-Kolchin M., Pawlowski M. S., Graus A. S., 2018, preprint (arXiv e-prints)

Kim J.-h. et al., 2014, ApJS, 210, 14

Kirby E. N., Bullock J. S., Boylan-Kolchin M., Kaplinghat M., Cohen J. G., 2014, MNRAS, 439, 1015

Kirby E. N., Rizzi L., Held E. V., Cohen J. G., Cole A. A., Manning E. M., Skillman E. D., Weisz D. R., 2017, ApJ, 834, 9

Klypin A., Kravtsov A. V., Valenzuela O., Prada F., 1999, ApJ, 522, 82

Knollmann S. R., Knebe A., 2011, AHF: Amiga's Halo Finder, Astrophysics Source Code Library, record ascl:1102.009

Koch A., Kleyna J. T., Wilkinson M. I., Grebel E. K., Gilmore G. F., Evans N. W., Wyse R. F. G., Harbeck D. R., 2007, AJ, 134, 566

Kroupa P., 2001, MNRAS, 322, 231

Krumholz M. R., Gnedin N. Y., 2011, ApJ, 729, 36

Larson D. et al., 2011, ApJS, 192, 16

Leaman R. et al., 2012, ApJ, 750, 33

Lee M. G., Yuk I.-S., Park H. S., Harris J., Zaritsky D., 2009, ApJ, 703, 692

Lewis G. F., Ibata R. A., Chapman S. C., McConnachie A., Irwin M. J., Tolstoy E., Tanvir N. R., 2007, MNRAS, 375, 1364

Martin N. F., de Jong J. T. A., Rix H.-W., 2008b, ApJ, 684, 1075

Martin N. F. et al., 2008a, ApJ, 672, L13

Martin N. F. et al., 2009, ApJ, 705, 758

Martin N. F. et al., 2013a, ApJ, 772, 15

Martin N. F. et al., 2013b, ApJ, 779, L10

Martínez-Delgado D., Gallart C., Aparicio A., 1999, AJ, 118, 862

Mateo M., Olszewski E. W., Morrison H. L., 1998, ApJ, 508, L55

Mateo M., Olszewski E. W., Walker M. G., 2008, ApJ, 675, 201

Ma X., Hopkins P. F., Faucher-Giguère C.-A., Zolman N., Muratov A. L., Kereš D., Quataert E., 2016, MNRAS, 456, 2140

McCall M. L., 2014, MNRAS, 440, 405

McConnachie A. W., 2012, AJ, 144, 4

McConnachie A. W., Irwin M. J., 2006, MNRAS, 365, 1263

McConnachie A. W., Irwin M. J., Ferguson A. M. N., Ibata R. A., Lewis G. F., Tanvir N., 2005, MNRAS, 356, 979

McConnachie A. W., Irwin M. J., Lewis G. F., Ibata R. A., Chapman S. C., Ferguson A. M. N., Tanvir N. R., 2004, MNRAS, 351, L94

McConnachie A. W. et al., 2008, ApJ, 688, 1009

Merritt D., Graham A. W., Moore B., Diemand J., Terzić B., 2006, AJ, 132, 2685

Monaco L., Bellazzini M., Ferraro F. R., Pancino E., 2004, MNRAS, 353, 874

Moore B., Ghigna S., Governato F., Lake G., Quinn T., Stadel J., Tozzi P., 1999, ApJ, 524, L19

More S., Diemer B., Kravtsov A. V., 2015, ApJ, 810, 36
Muratov A. L., Kereš D., Faucher-Giguère C.-A., Hopkins P. F., Quataert E., Murray N., 2015, MNRAS, 454, 2691

Muñoz R. R. et al., 2005, ApJ, 631, L137

Navarro J. F., Frenk C. S., White S. D. M., 1996, ApJ, 462, 563

Oman K. A., Navarro J. F., Sales L. V., Fattahi A., Frenk C. S., Sawala T., Schaller M., White S. D. M., 2016, MNRAS, 460, 3610

Oman K. A. et al., 2015, MNRAS, 452, 3650

Oñorbe J., Boylan-Kolchin M., Bullock J. S., Hopkins P. F., Kereš D., Faucher-Giguère C.-A., Quataert E., Murray N., 2015, MNRAS, 454, 2092

Oñorbe J., Garrison-Kimmel S., Maller A. H., Bullock J. S., Rocha M., Hahn O., 2014, MNRAS, 437, 1894

Oñorbe J., Hennawi J. F., Lukić Z., 2017, ApJ, 837, 106

Papastergis E., Giovanelli R., Haynes M. P., Shankar F., 2015, A\&A, 574, A113

Papastergis E., Shankar F., 2016, A\&A, 591, A58

Patel E., Besla G., Mandel K., Sohn S. T., 2018, ApJ, 857, 78

Perez F., Granger B. E., 2007, Comput. Sci. Eng., 9, 21

Peñarrubia J., Benson A. J., Walker M. G., Gilmore G., McConnachie A. W., Mayer L., 2010, MNRAS, 406, 1290

Peñarrubia J., McConnachie A. W., Navarro J. F., 2008, ApJ, 672, 904

Pietrzyński G., Górski M., Gieren W., Ivanov V. D., Bresolin F., Kudritzki R.-P., 2009, AJ, 138, 459

Pietrzyński G. et al., 2008, AJ, 135, 1993

Planck Collaboration XIII, 2016, A\&A, 594, A13

Pontzen A., Governato F., 2012, MNRAS, 421, 3464

Power C., Navarro J. F., Jenkins A., Frenk C. S., White S. D. M., Springel V., Stadel J., Quinn T., 2003, MNRAS, 338, 14

Price D. J., Monaghan J. J., 2007, MNRAS, 374, 1347

Richardson J. C. et al., 2011, ApJ, 732, 76

Salomon J.-B., Ibata R. A., Famaey B., Martin N. F., Lewis G. F., 2016, MNRAS, 456, 4432

Samuel J. et al., 2019, MNRAS, preprint (arXiv:1904.11508)

Sanderson R. E. et al., 2017, ApJ, 869, 12

Saviane I., Held E. V., Piotto G., 1996, A\&A, 315, 40

Sawala T., Pihajoki P., Johansson P. H., Frenk C. S., Navarro J. F., Oman K. A., White S. D. M., 2017, MNRAS, 467, 4383

Sawala T. et al., 2016a, MNRAS, 456, 85

Sawala T. et al., 2016b, MNRAS, 457, 1931

Schaye J. et al., 2015, MNRAS, 446, 521

Sick J., Courteau S., Cuillandre J.-C., Dalcanton J., de Jong R., McDonald M., Simard D., Tully R. B., 2015, in Cappellari M., Courteau S., eds, Proc. IAU Symp. 311, Galaxy Masses as Constraints of Formation Models. Kluwer, Dordrecht, p. 82

Simon J. D., Geha M., 2007, ApJ, 670, 313

Simon J. D., Prada F., Vílchez J. M., Blitz L., Robertson B., 2006, ApJ, 649, 709

Simpson C. M., Grand R. J. J., Gómez F. A., Marinacci F., Pakmor R., Springel V., Campbell D. J. R., Frenk C. S., 2018, MNRAS, 478, 548

Somerville R. S., 2002, ApJ, 572, L23

Sparre M., Hayward C. C., Feldmann R., Faucher-Giguère C.-A., Muratov A. L., Kereš D., Hopkins P. F., 2017, MNRAS, 466, 88

Springel V., 2005, MNRAS, 364, 1105

Springel V. et al., 2005, Nature, 435, 629

Stanimirović S., Staveley-Smith L., Jones P. A., 2004, ApJ, 604, 176

Stinson G., Seth A., Katz N., Wadsley J., Governato F., Quinn T., 2006, MNRAS, 373, 1074

Su K.-Y., Hopkins P. F., Hayward C. C., Faucher-Giguère C.-A., Kereš D., Ma X., Robles V. H., 2017, MNRAS, 471, 144

Teyssier M., Johnston K. V., Kuhlen M., 2012, MNRAS, 426, 1808

Tikhonov N. A., Galazutdinova O. A., 2009, Astron. Lett., 35, 748

Tollerud E. J., Boylan-Kolchin M., Barton E. J., Bullock J. S., Trinh C. Q., 2011, ApJ, 738, 102

Tollerud E. J., Boylan-Kolchin M., Bullock J. S., 2014, MNRAS, 440, 3511 Tollerud E. J., Bullock J. S., Strigari L. E., Willman B., 2008, ApJ, 688, 277 Tollerud E. J., Geha M. C., Vargas L. C., Bullock J. S., 2013, ApJ, 768, 50 Tollerud E. J. et al., 2012, ApJ, 752, 45 
Turk M. J., Smith B. D., Oishi J. S., Skory S., Skillman S. W., Abel T., Norman M. L., 2011, ApJS, 192, 9

Udalski A., Szymanski M., Kubiak M., Pietrzynski G., Soszynski I., Wozniak P., Zebrun K., 1999, Acta Astron., 49, 201

van den Bosch F. C., Ogiya G., 2018, MNRAS, 475, 4066

van der Marel R. P., Alves D. R., Hardy E., Suntzeff N. B., 2002, AJ, 124, 2639

van der Marel R. P., Fardal M., Besla G., Beaton R. L., Sohn S. T., Anderson J., Brown T., Guhathakurta P., 2012, ApJ, 753, 8

van der Marel R. P., Kallivayalil N., 2014, ApJ, 781, 121

van der Marel R. P., van den Bosch F. C., 1998, AJ, 116, 2220

van der Walt S., Colbert S. C., Varoquaux G., 2011, Comput. Sci. Eng., 13, 22

van Dokkum P. G., Abraham R., Merritt A., Zhang J., Geha M., Conroy C., 2015, ApJ, 798, L45

Walker M. G., Mateo M., Olszewski E. W., 2009, AJ, 137, 3100

Weiner B. J. et al., 2006, ApJ, 653, 1027

Wetzel A. R., Hopkins P. F., Kim J.-h., Faucher-Giguère C.-A., Kereš D., Quataert E., 2016, ApJ, 827, L23

Wilcots E. M., Miller B. W., 1998, AJ, 116, 2363

Wolf J., Martinez G. D., Bullock J. S., Kaplinghat M., Geha M., Muñoz R. R., Simon J. D., Avedo F. F., 2010, MNRAS, 406, 1220

Woo J., Courteau S., Dekel A., 2008, MNRAS, 390, 1453

Yang S.-C., Sarajedini A., 2012, MNRAS, 419, 1362

Zolotov A. et al., 2012, ApJ, 761, 71

\section{APPENDIX A: OBSERVATIONAL CATALOGUE}

Table A1 lists the properties of the galaxies plotted in Figs 2, 4, 5, and 6, separated in the MW, M31, and Local Field subsamples. The columns list the distance from the MW and M31, adopted stellar mass, 3D half-light radius, line-of-sight velocity dispersion, the implied circular velocity at $R_{1 / 2}$, and references. Galaxies without an entry for $\left(R_{1 / 2}, V_{1 / 2}\right)$ are not included in Figs 4 and 5, and galaxies without an entry for $\sigma_{*}$ are not included in
Fig. 6. While we include the properties of M32 as listed in Tollerud et al. (2014), we remind the reader that it falls outside the bounds of our plots in Figs 4, 5, and 6. Dynamical values $\left(R_{1 / 2}\right.$ and $\left.V_{1 / 2}\right)$ adopted from Wolf et al. (2010) were calculated using data from Walker, Mateo \& Olszewski (2009) along with Muñoz et al. (2005), Koch et al. (2007), Simon \& Geha (2007), and Mateo, Olszewski \& Walker (2008).

The references in the last column are as follows: (1) de Vaucouleurs et al. (1991); (2) Clementini et al. (2003); (3) van der Marel et al. (2002); (4) Udalski et al. (1999); (5) Harris \& Zaritsky (2006); (6) Monaco et al. (2004); (7) Mateo, Olszewski \& Morrison (1998); (8) Frinchaboy et al. (2012); (9) Pietrzyński et al. (2009); (10) Wolf et al. (2010); (11) Bellazzini et al. (2004); (12) Pietrzyński et al. (2008); (13) Bellazzini, Gennari \& Ferraro (2005); (14) Lee et al. (2009); (15) Carrera et al. (2002); (16) Bonanos et al. (2004); (17) Martin et al. (2008a); (18) McConnachie et al. (2005); (19) Simon et al. (2006); (20) Geha et al. (2006); (21) Fiorentino et al. (2010); (22) Howley et al. (2012); (23) Geha et al. (2010); (24) Tikhonov \& Galazutdinova (2009); (25) Wilcots \& Miller (1998); (26) McConnachie \& Irwin (2006); (27) Tollerud et al. (2012); (28) Martin et al. (2013a); (29) Conn et al. (2012); (30) Ho et al. (2012); (31) Richardson et al. (2011); (32) Collins et al. (2013); (33) Martin et al. (2009); (34) Cook et al. (1999); (35) Ibata et al. (2007); (36) McConnachie et al. (2008); (37) Brasseur et al. (2011); (38) Bell, Slater \& Martin (2011); (39) Tollerud et al. (2013); (40) Collins et al. (2010); (41) Yang \& Sarajedini (2012); (42) Chapman et al. (2013); (43) Bernard et al. (2010); (44) Kirby et al. (2014); (45) Hunter \& Elmegreen (2006); (46) Gieren et al. (2006) (47) Dale et al. (2007); (48) Leaman et al. (2012); (49) Bernard et al. (2009); (50) Kirby et al. (2017); (51) Martin et al. (2013b); (52) MartínezDelgado, Gallart \& Aparicio (1999); (53) Saviane, Held \& Piotto (1996); (54) Fraternali et al. (2009); (55) de Jong et al. (2008); (56) Simon \& Geha (2007). 
Table A1. Observational properties of the galaxies included in our sample: distance from the MW and M31, adopted stellar mass, position in $V_{\text {circ }}(r)$ space, and line-of-sight stellar velocity dispersion. References are listed in Appendix A.

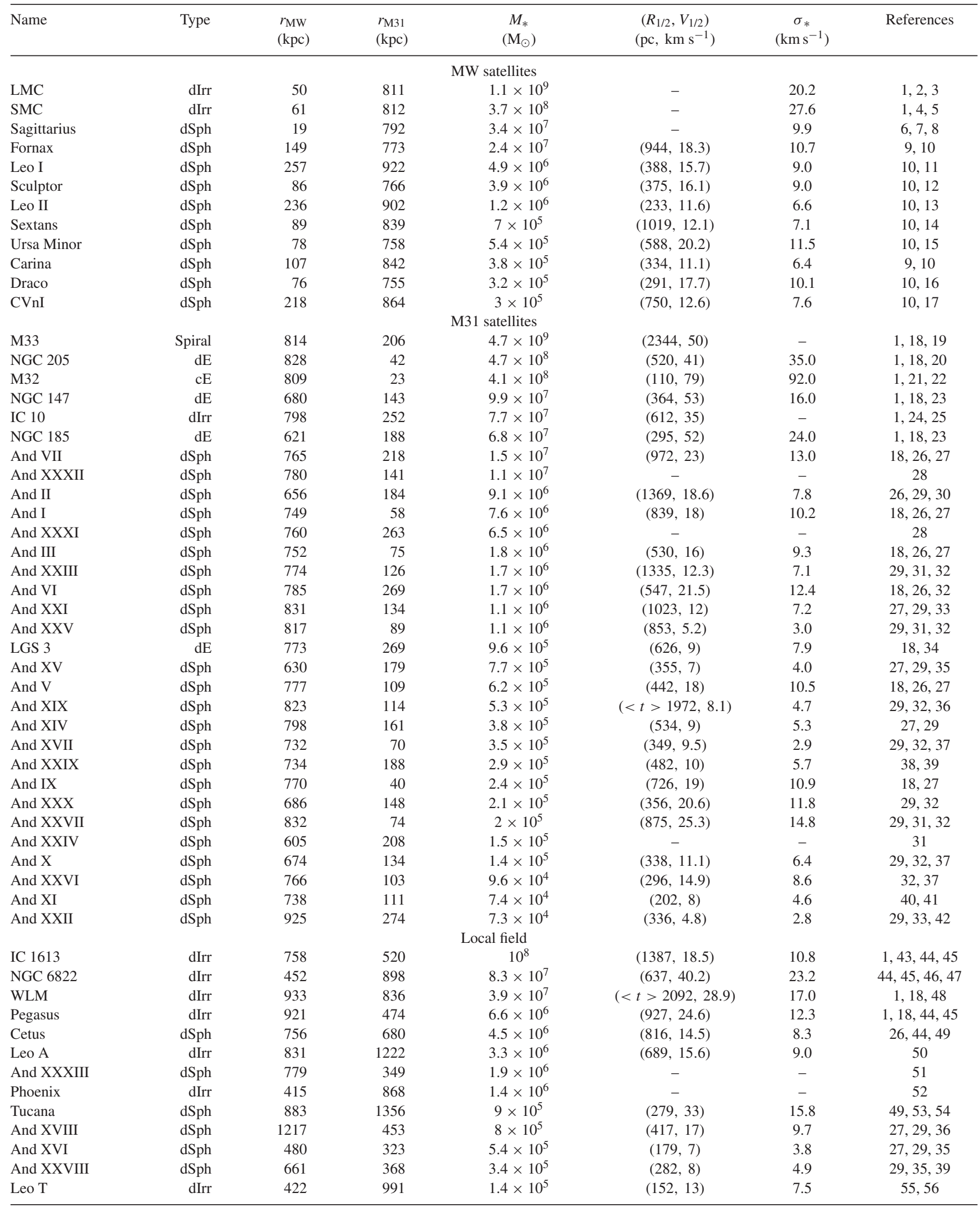




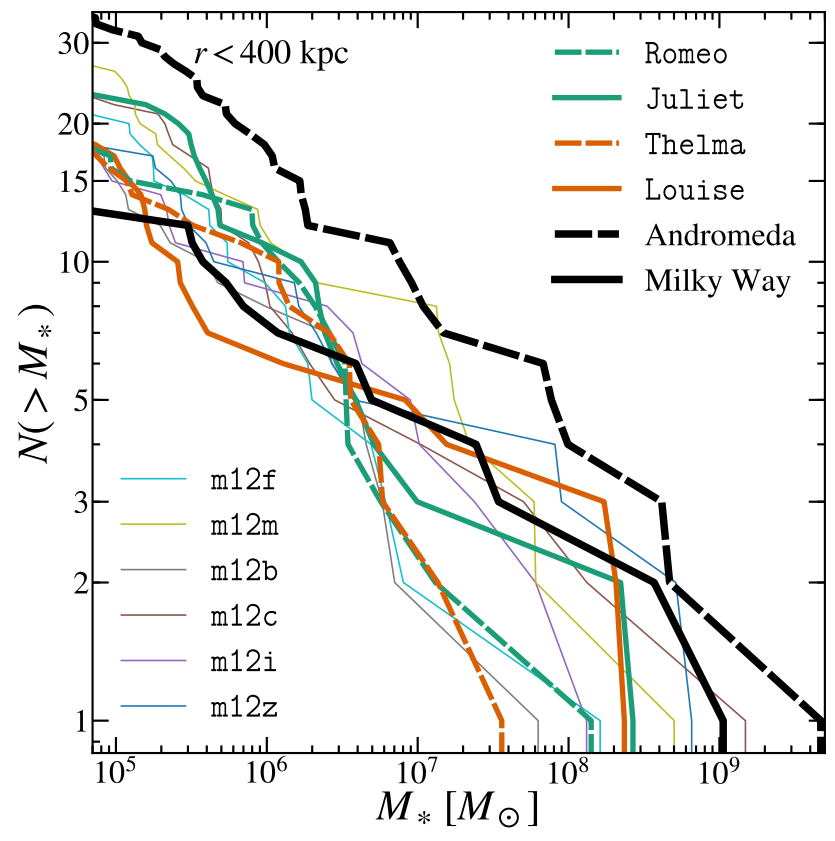

Figure B1. Identical to the left-hand panel of Fig. 2, but counting galaxies within $400 \mathrm{kpc}$ (rather than $300 \mathrm{kpc}$ ). Several of the simulations (particularly $\mathrm{m} 12 \mathrm{~m}$ ) are slightly closer to the SMF of M31 if satellites are defined as $r<$ $400 \mathrm{kpc}$, and this also reduces the tension with the SMF (Fig. 2, right-hand panel) and TBTF comparison (Fig. 5, right-hand panel) of the Local Field, by reassigning a few haloes to M31. The runs still tend to underpredict the SMF of M31, however

\section{APPENDIX B: SATELLITE GALAXIES WITHIN $400 \mathrm{KPC}$}

In the main text, we select $r=300 \mathrm{kpc}$ as the dividing radius between satellites and non-satellite galaxies. However, this is motivated primarily by historical reasons, and is somewhat arbitrary; while the virial radius of these hosts ranges from $\simeq 240$ to $300 \mathrm{kpc}$, the virial radius does not have an intrinsic physical meaning. Instead, the physical boundary of the halo is more closely related to the splashback radius, which is typically $\simeq 0.8-1 \times R_{200 \mathrm{~m}}$, the radius that encloses an average of 200 times the background density (More, Diemer \& Kravtsov 2015). For our hosts, $R_{200 \mathrm{~m}} \simeq 310$ $380 \mathrm{kpc}$. Moreover, there are curious gaps of known satellites at 257-415 kpc around the MW and 274-349 kpc around M31 (Samuel et al., in preparation). Therefore, Fig. B1 follows the lefthand panel of Fig. 2 in counting galaxies around each host, but here counts dwarf galaxies within $400 \mathrm{kpc}$. The exact numbers shift slightly, but the overall conclusion that our present sample of hosts (with $M_{\text {vir }} \leq 1.45 \times 10^{12} \mathrm{M}_{\odot}$ ) underproduces the SMF of M31 is unchanged. This does, however, somewhat reduce both this tension and the apparent tension with the Local Field SMF and TBTF problems, by reassigning $\sim 5-10$ haloes with $M_{*} \sim 10^{5}-10^{6} \mathrm{M}_{\odot}$ from the Local Field to M31.

\section{APPENDIX C: IMPACT OF DENSITY PROFILES ON CIRCULAR VELOCITIES}

In Section 5, we compute circular velocity profiles for galaxies in the hydrodynamic simulations by first fitting the resolved portion of the halo with an $(\alpha, \beta, \gamma)$ density profile:

$\rho(r)=\rho_{0}\left(\frac{r}{r_{\mathrm{s}}}\right)^{-\gamma}\left[1+\left(\frac{r}{r_{\mathrm{s}}}\right)^{\alpha}\right]^{-(\beta-\gamma) / \alpha}$.

Here, we examine the impact of using the interpolated density profile to compute the central mass versus using the raw particle data, as well as the effect of varying $r_{\min }$, the smallest radius used in fitting $\rho(r)$, and the radius within which we compute $M(r)$ from the fits. Fig. C1 shows the circular velocity profiles for three satellite galaxies of Juliet obtained from the raw particle data and from fits with varying $r_{\min }$. Following Hopkins et al. (2018, and also see Power et al. 2003), we determine $r_{\min }$ as the radius that contains a given number of dark matter particles. Fig. C1 illustrates that the correction to the circular velocity from underresolving the central $\sim 100-500 \mathrm{pc}$ is typically only $\lesssim 3 \mathrm{~km} \mathrm{~s}^{-1}$ at $500 \mathrm{pc}$ and is nearly negligible at $1 \mathrm{kpc}$, regardless of the number of DM particles used to define $r_{\text {min }}$. The lone exception, the fit with $r_{\text {min }}$ determined by $N_{\text {enc }}=2200$ in the right-hand panel, diverges because the implied $r_{\min }$ is comparable to $R_{\max }$, resulting in a poor fit to the density profile. To emphasize that our TBTF counts are insensitive to the inner profiles, such that we can still match constraints on the MW dSphs with cuspy central densities, we additionally plot NFW profiles normalized to $R_{\max }$ and $V_{\max }$. The implied $V_{\text {circ }}$ profiles can vary by a factor of $\sim 2$ at $250 \mathrm{pc}$ in subhaloes with higher $M_{*}$ (where supernova feedback is more important), but a combination of overall mass-loss from surviving subhaloes and enhanced subhalo destruction from the central galaxy (relative to the DMO simulations) is nearly sufficient to explain TBTF.

In order to explicitly demonstrate that our assumption of NFW profiles for the DMO simulations does not affect our overall results, Fig. C2 shows the circular velocity profiles for Romeo \& Juliet, but here using the raw particle data. Even without correcting for the effects of gravitational softening or assuming a density profile, the DMO simulations contain $\sim 15$ subhaloes that can only host Draco and Ursa Minor. 


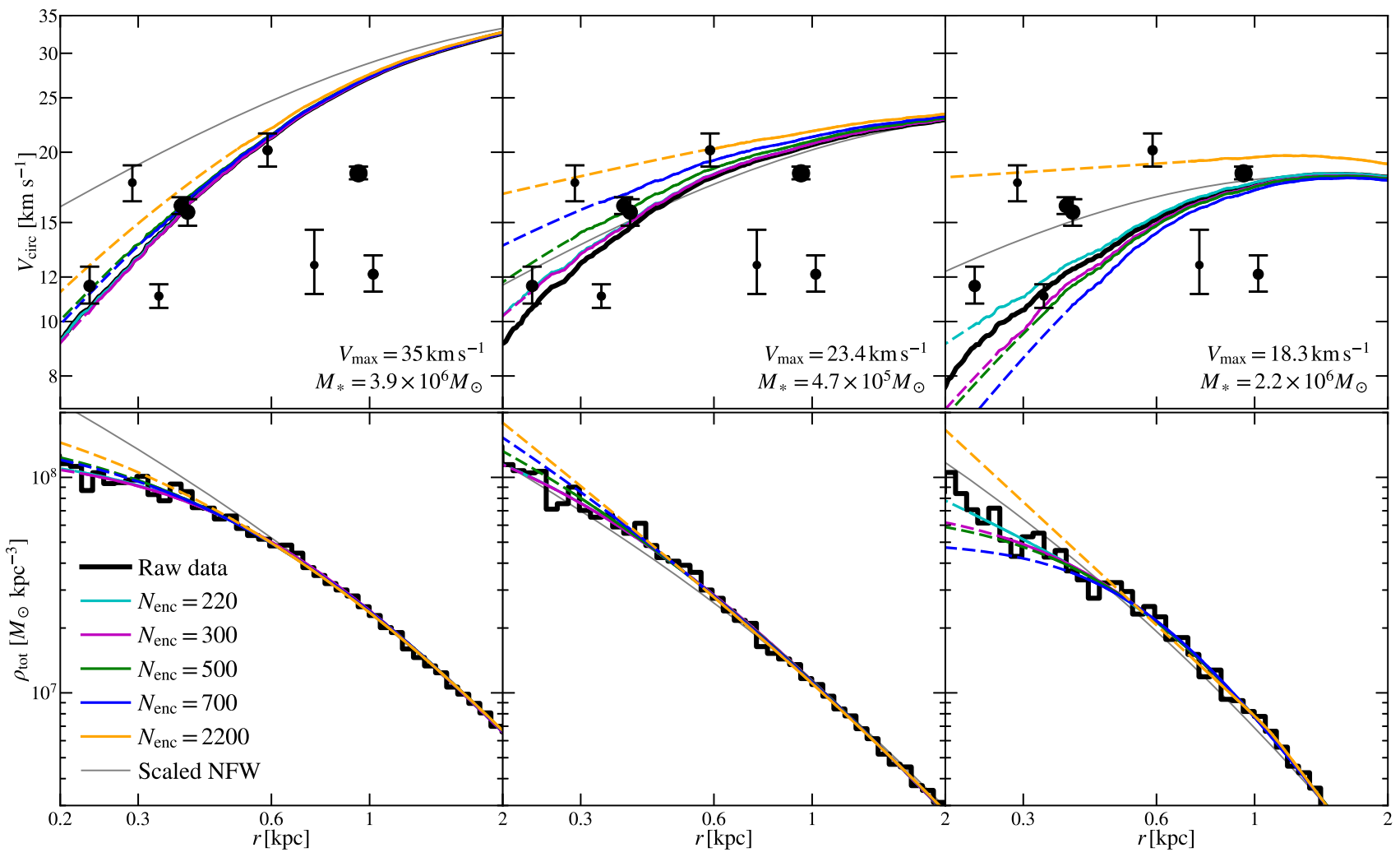

Figure C1. Circular velocity curves (top) and density profiles (bottom) of three satellites around Juliet from the raw particle data, from extrapolating fitted density profiles inside the radius enclosing $N_{\text {enc }}$ dark matter particles, and from assuming a NFW profile based on $R_{\max }$ and $V_{\max }$, together with the usual constraints on the MW dSphs. The coloured lines become dashed at $r<r_{\min }$, i.e. where $V_{\text {circ }}$ is entirely determined by the fits. The effects of varying $N_{\text {enc }}$ are negligible, particularly in the context of TBTF, provided that the implied minimum radius is small enough to capture the curvature of the density profile.

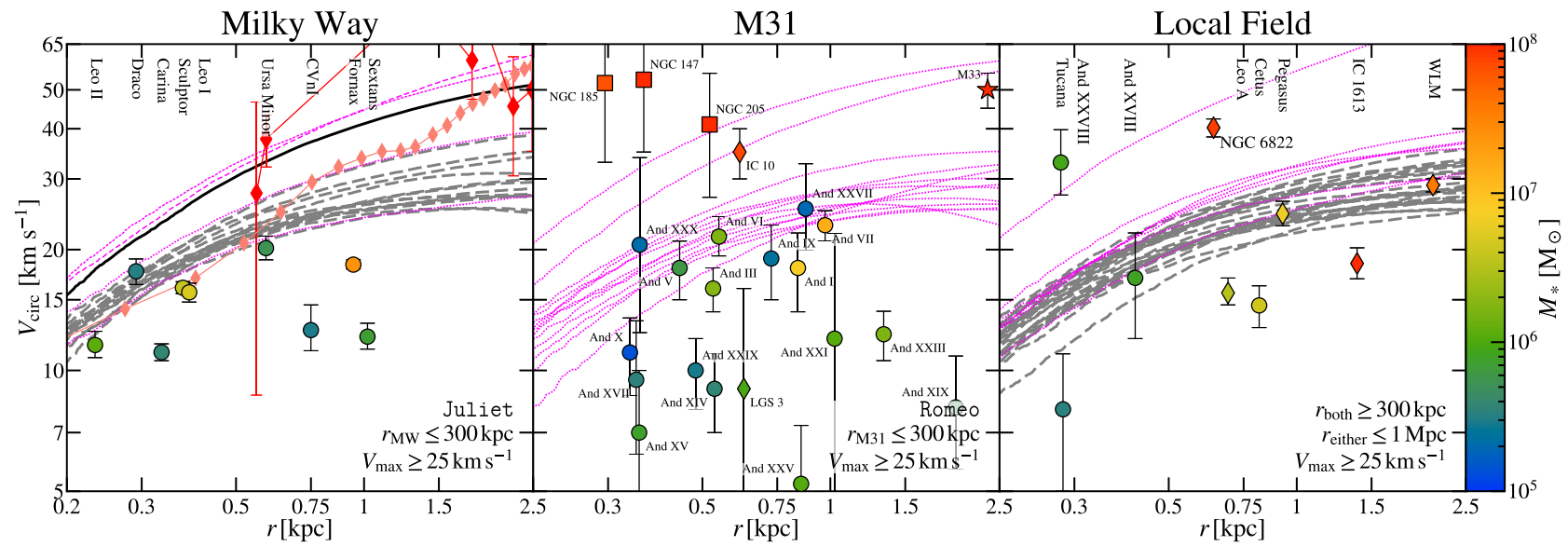

Figure C2. Identical to the top panels of Fig. 4, but here using the raw particle data for the DMO simulations. The conclusions are unchanged: the DMO simulations contain a wealth of subhaloes with circular velocities at $r \sim 300-1500 \mathrm{pc}$ that are incompatible with the MW dSphs and the majority of the M31 satellites.

This paper has been typeset from a $\mathrm{T}_{\mathrm{E}} \mathrm{X} / \mathrm{LT} \mathrm{E} \mathrm{X}$ file prepared by the author. 